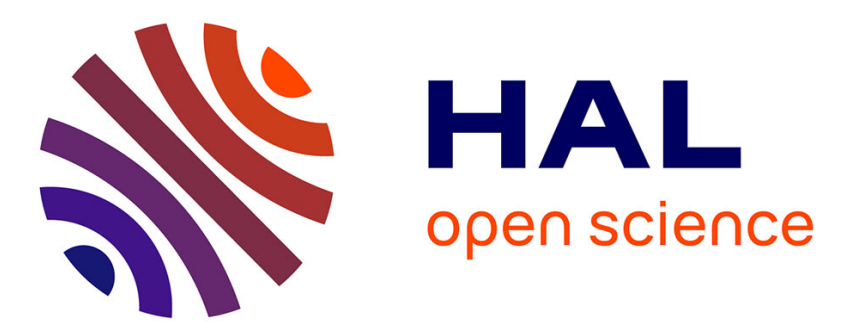

\title{
Prise en charge endoscopique des fistules gastriques après sleeve gastrectomy par prothèses double queue de cochon : technique BARTOLI \\ Constance Deschepper
}

\section{- To cite this version: \\ Constance Deschepper. Prise en charge endoscopique des fistules gastriques après sleeve gastrectomy par prothèses double queue de cochon: technique BARTOLI. Hépatologie et Gastroentérologie. 2017. dumas-01710841}

\section{HAL Id: dumas-01710841 https://dumas.ccsd.cnrs.fr/dumas-01710841}

Submitted on 27 Apr 2018

HAL is a multi-disciplinary open access archive for the deposit and dissemination of scientific research documents, whether they are published or not. The documents may come from teaching and research institutions in France or abroad, or from public or private research centers.
L'archive ouverte pluridisciplinaire $\mathbf{H A L}$, est destinée au dépôt et à la diffusion de documents scientifiques de niveau recherche, publiés ou non, émanant des établissements d'enseignement et de recherche français ou étrangers, des laboratoires publics ou privés. 


\title{
Prise en charge endoscopique des fistules
}

gastriques après sleeve gastrectomy par prothèses

\section{double queue de cochon : Technique BARTOLI.}

\author{
THESE POUR LE DOCTORAT EN \\ MEDECINE-HEPATOGASTROENTEROLOGIE \\ (DIPLOME D'ETAT) \\ PRESENTEE ET SOUTENUE PUBLIQUEMENT \\ LE VENDREDI 17 MARS 2017 \\ PAR Constance DESCHEPPER
}

PRESIDENT DU JURY : Monsieur le Professeur NGUYEN-KHAC

MEMBRES DU JURY : Monsieur le Professeur REGIMBEAU

Madame le Professeur DESAILLOUD

Monsieur le Docteur MAHJOUB

Monsieur le Docteur HAKIM 
A mon Maître,

Monsieur le Professeur Eric NGUYEN-KHAC

Professeur des Universités-Praticien Hospitalier

(Hépato-Gastroentérologue)

Chef du Service d'Hépato-Gastroentérologie

Pôle "Médico-chirurgical digestif, rénal, infectieux, médecine interne et endocrinologie" (D.R.I.M.E)

Vous me faîtes l'honneur de juger ce travail, et je vous en remercie sincèrement.

Votre expertise et votre rigueur sont à prendre en modèle.

Veuillez trouver ici l'expression de mon respect et de ma reconnaissance pour votre enseignement. 
A mon Maître,

Monsieur le Professeur Jean-Marc REGIMBEAU

Professeur des Universités-Praticien Hospitalier

(Chirurgie digestive)

Responsable du service de chirurgie digestive

Pôle "Médico-chirurgical digestif, rénal, infectieux, médecine interne et endocrinologie" (D.R.I.M.E)

Merci de me faire l'honneur et le plaisir de juger ce travail.

J'ai pu apprécier vos qualités pédagogiques à chaque réunion multidisciplinaire.

Veuillez trouver ici l'expression de ma gratitude et de mon profond respect. 
A mon Maître,

Madame le Professeur Rachel DESAILLOUD

Professeur des Universités-Praticien Hospitalier

(Endocrinologie, Diabétologie et Maladies Métaboliques)

Vous me faîtes l'honneur de juger ce travail, et je vous en remercie sincèrement.

Veuillez trouver ici l'expression de ma gratitude et de mon profond respect. 
A mon Maître,

Monsieur le Docteur Yazine MAHJOUB

Maître de Conférences des Universités - Praticien Hospitalier

Anesthésiologie réanimation, médecine d'urgence

Merci de me faire l'honneur de juger ce travail.

Ton expertise, ta rigueur et ta disponibilité sont autant de choses à prendre en modèle. 
A mon Maître,

Monsieur le Docteur Sami HAKIM

Praticien hospitalier (hépato-gastro-entérologie)

Merci pour la confiance que tu m'as accordée.

Merci pour ta disponibilité, ta gentillesse et tout ce que tu m'as et vas encore m'apprendre. 


\section{Remerciements :}

Au Docteur Bartoli, que je n'ai pas eu la chance de connaitre, qui est l'initiateur de cette technique et à qui je dois ce travail.

Au Professeur Dupas, Docteur Joly, Docteur Delcenserie et Docteur Yzet.

Merci pour votre disponibilité, votre gentille et votre implication à nous former.

A Franck, merci pour la confiance que tu nous accordes, l'expérience que tu nous transmets et ton humour qui te caractérise si bien.

Au Docteur Rebibo, merci pour ton aide sans qui ce travail n'aurait pu être réalisé.

A mes anciens chefs de clinique :

- Mathurin, merci pour ton accompagnement durant mes premiers pas hasardeux d'interne, ta disponibilité et ton aide précieuse tout au long de l'internat.

- Marthe, merci pour ta gentillesse et tes coups de pouce à Abbeville et aux endoscopies.

- Henri, merci pour ta disponibilité, tes gentils sarcasmes, et ton enseignement.

A mes chefs : Justine, Adrien, Morgane, Vincent, Pierre

Merci pour vos conseils, votre esprit d'équipe et tout ce que vous m'avez appris. 
A mes Co internes et jeunes chefs de choc, sans qui l'internat n'aurait pas eu toutes ses couleurs, ses fous rires et ses verres de trop!

Marion, notre semestre en A5 sud est à l'origine d'une belle amitié.

Clara, on continuera à refaire le monde même à Lille.

Julien, merci d'avoir assuré la présence masculine et la sécurité de tes co-internes lors des soirées DES.

Clémentine, toujours disponible pour les potins, tubas ou verres de vin.

Aline merci pour tes conseils et ta gentillesse.

Justine, Valérie, Marie, Jean-Phi et Rux merci pour vos précieux conseils ses derniers mois.

Et à mes jeunes co-internes, Anouck, Clément Xixi, Neila, Julie, Marion et Carole.

A toute l'équipe du service de gastroentérologie, des endoscopies, Catherine et Louisette, qui m'ont permis de passer un super internat.

A toute l'équipe du service d'hépato-gastroentérologie d'Abbeville.

A toute l'équipe du service de réanimation chirurgicale du CHU d'Amiens. 
Au Professeur Hautecoeur, doyen de la faculté de médecine et de maïeutique de Lille.

Au Professeur Forzy, ancien doyen de la faculté de médecine et de maïeutique de Lille.

Au Professeur Lucidarme et à toute l'équipe du service d'hépato-gastroentérologie de SaintPhilibert. 
A Thibault,

Merci pour ta patience, ton optimisme et ton humour, toi pour qui l'internat fut long.

A Papa et Maman,

Merci pour tout.

Merci de m'avoir encouragée, soutenue et fait de moi ce que je suis aujourd'hui.

A Victoire et Edouard (Wichky et Eddy),

Parce qu'elle vous a soulée votre petite sœur avec ses cours de médecine, merci d'être présents avec Hugues, Marie et Jeanne aujourd'hui.

A mon grand-père, Professeur Jacques Crinquette.

A mon oncle, Docteur Jacques-François Crinquette, merci de m'avoir donné l'envie et le goût de devenir ta consœur. Je te suis reconnaissante de m'avoir transmis si gentiment ton expérience.

Et sans oublier les coupines et la glieglebluck T. 
Liste des abréviations utilisées :

AG : anneau gastrique

HTA : hypertension artérielle

IMC : indice de masse corporelle

IPP : inhibiteurs de la pompe à proton

OTSC : over-the scope clip

PAC : port-à-cathéter

PDQC : prothèses double queue de cochon

P1: période 1

P2 : période 2

PT : période totale

SAOS : syndrome d'apnée obstructive du sommeil

SEMS : self-expandable metallic stent

SG : sleeve gastrectomy

SNJ : sonde naso jéjunale

SRIS : syndrome de réponse inflammatoire systémique 


\section{TABLE DES MATIERES}

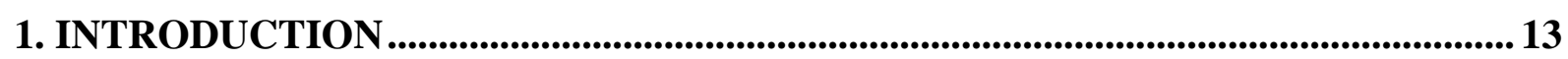

1.1 Epidémiologie ..........................................................................................................13

1.2 Complications de l'obésité ................................................................................. 13

1.3 Impact médico-économique de l'obésité ................................................................. 16

1.4 Traitement chirurgical et endoscopique de l'obésité................................................ 16

1.5. Objectif de l'étude

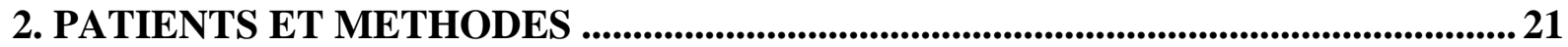

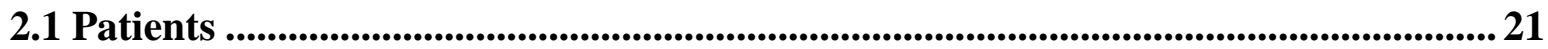

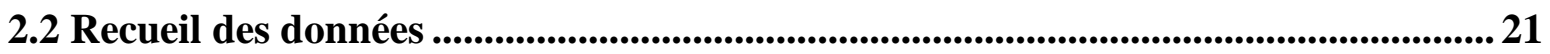

2.3 Procédure de traitement des fistules gastriques post sleeve gastrectomy ................ 22

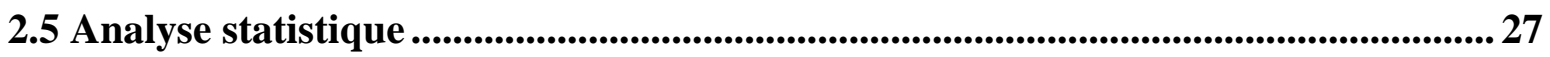

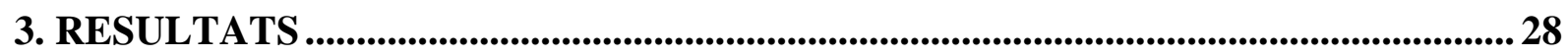

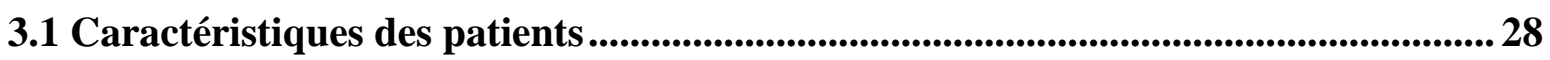

3.2 Efficacité de la technique Bartoli ..................................................................... 30

3.3 Efficacité des traitements endoscopiques ............................................................................ 30

3.4 Evaluation des modalités de la technique Bartoli pour la période PT ..................... 31

3.4.1 Evaluation des modalités du drainage externe ...................................................... 31

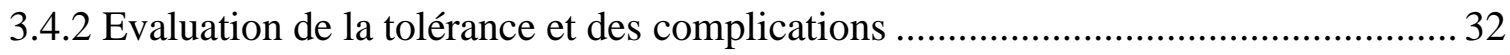

3.4.3 Evaluation des modalités endoscopiques, d'alimentation, d'hospitalisation........... 32

3.5 Evaluation de la technique Bartoli pour les périodes P1 et P2, (Tableau 3) ............ 33

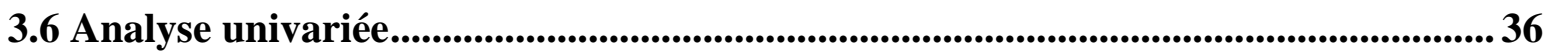

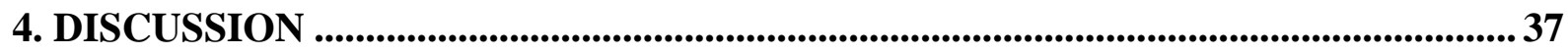

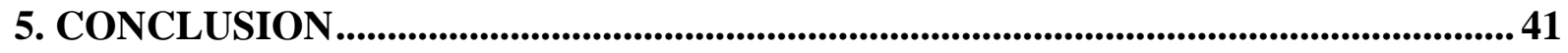

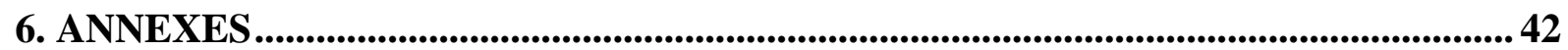

7. REFERENCES BIBLIOGRAPHIQUES ............................................................ 48 


\section{INTRODUCTION}

\subsection{Epidémiologie}

L'obésité est définie par un indice de masse corporelle (IMC $=$ poids $[\mathrm{kg}] /$ taille $\left[\mathrm{m}^{2}\right]$ ) supérieur à $30 \mathrm{~kg} / \mathrm{m}^{2}$, et dans sa forme abdominale par un tour de taille supérieur à $88 \mathrm{~cm}$ chez les femmes et $102 \mathrm{~cm}$ chez les hommes.

Dans le monde, plus de 1.9 milliards d'adultes de plus de 18 ans étaient en surpoids dont 600 millions d'obèses d'après les dernières mesures épidémiologiques de l'organisation mondiale de la santé en 2014. En France, l'obésité concerne 6.9 millions de personnes et sa prévalence était estimée à $15 \%$ en 2012 contre $14.5 \%$ en 2009 (1).

L'obésité expose à de nombreuses complications notamment cardiovasculaires, métaboliques, respiratoires, ostéo-articulaires, digestives ou encore néoplasiques ainsi qu'à une surmortalité doublée par rapport à la population générale, principalement de cause cardiovasculaire mais aussi par cancer $(2,3)$.

\subsection{Complications de l'obésité :}

\section{- Complications métaboliques}

Parmi les complications métaboliques, le diabète non insulinodépendant est la pathologie la plus fortement associée à l'obésité. Son incidence est six fois plus élevée que chez les sujets d'IMC normal. En France, les données de l'enquête Obépi 2012 estimait le nombre de personnes obèses ayant un diabète de type 2 à $13.5 \%$ (4). Cette pathologie a un réel impact avec une augmentation de la mortalité à 5 ans de $45 \%$ dans cette population, principalement de cause cardiovasculaire (5). Au niveau physiopathologique, l'obésité entraîne une diminution de la sensibilité à l'insuline qui constitue, avec la défaillance de la sécrétion d'insuline, l'un des deux facteurs pathogéniques principaux de ce type de diabète.

Une augmentation des triglycérides et une diminution du cholestérol-HDL sont observées lorsque l'IMC augmente. Chez les sujets les plus jeunes, on observe également une augmentation du cholestérol total, non-HDL et LDL (6). La physiopathologie de ces anomalies est caractérisée par un excès de production ou un défaut de catabolisme des lipoprotéines riches en triglycérides. L'excès de lipoprotéines riches en triglycérides est 
associé à d'autres anomalies, telles que l'augmentation de la lipémie postprandiale, la baisse du cholestérol-HDL et la présence de petites LDL denses, particulièrement athérogènes.

\section{- Complications cardio-vasculaires}

L'impact cardio-vasculaire de l'obésité est la principale source de morbi-mortalité. Le risque relatif de coronaropathie chez les sujets obèses comparés aux sujets de corpulence normale (IMC $<25 \mathrm{~kg} / \mathrm{m}^{2}$ ) est estimé à 1,7 chez les hommes et 3,1 chez les femmes. Le risque relatif d'hypertension artérielle (HTA) est estimé à 1,8 chez les hommes et 2,4 chez les femmes, le risque relatif d' insuffisance cardiaque est estimé à 1,8; comparable chez les hommes et chez les femmes (7) et le risque relatif d'accident vasculaire cérébral est évalué à 1,5 chez les sujets obèses $(8,9,10)$.

\section{- Complications néoplasiques}

L'obésité est responsable d'une augmentation de l'incidence de nombreux cancers, au premier rang desquels les cancers hormonodépendants (chez la femme : cancer de l'endomètre, de l'ovaire et du sein après la ménopause ; chez l'homme : cancer de la prostate) et les cancers digestifs (colorectal, œsophage, estomac). Il faut également citer les cancers du pancréas, du foie, du rein et de la thyroïde, les leucémies et le myélome multiple $(11,8)$.

\section{- Complications respiratoires}

L'obésité est responsable d'altérations de la mécanique ventilatoire, d'un syndrome restrictif, de modifications des échanges gazeux, se traduisant par une insuffisance respiratoire restrictive et par un syndrome d'apnée obstructive du sommeil (SAOS) dont l'incidence dans l'obésité est estimé entre 65 et $75 \%$ (12).

\section{- Complications ostéo-articulaires}

L'obésité peut être un facteur déclenchant ou aggravant de nombreuses affections dégénératives de l'appareil locomoteur telles que l'arthrose, en particulier la gonarthrose ou la coxarthrose, et la pathologie péri-articulaire. $(13,14)$. Les douleurs rachidiennes sont fréquentes et l'ensemble de ces anomalies favorisent la sédentarité et l'inactivité physique de ces patients (15). La gonarthrose et la pathologie rachidienne mécanique sont les principales causes d'altération de leur qualité de vie et de handicap. Les sujets obèses ont un risque près de trois fois supérieur de prendre une retraite précoce en raison de douleurs lombaires chroniques par rapport aux sujets de corpulence normale (8). Une de perte de poids de $10 \%$ 
associée à la pratique d'une activité physique de résistance et d'endurance permettent une réduction des douleurs et une amélioration des capacités fonctionnelles $(12,13)$.

\section{- Complications hépatobiliaires}

La stéatose hépatique est essentiellement liée à l'obésité abdominale associée à une dyslipidémie et une insulinorésistance. La stéatose peut évoluer vers la fibrose hépatique, la cirrhose et le carcinome hépatocellulaire (16).

La pathologie biliaire lithiasique est plus fréquente chez les sujets obèses que chez les sujets de poids normal, en particulier chez les femmes; le risque relatif varie de deux à six selon les études $(8,17)$.

\section{- Complications réno-vésicales}

L'obésité augmente d'un facteur de 1,7 à 2,4 la prévalence de l'incontinence urinaire d'effort associée à la gravité de l'obésité (18). L'élévation de la pression intra-abdominale augmente les contraintes de pression sur la vessie et sur le plancher pelvien, et est responsable d'altérations de la mobilité urétrale, entrainant des incontinences urinaires d'effort et/ou des impériosités

L'obésité est associée à une augmentation du risque d'atteinte rénale chronique, de protéinurie et d'insuffisance rénale, dont l'incidence est variable (19). Indépendamment de l'insulinorésistance et de l'hypertension, l'obésité pourrait avoir des effets propres sur le rein comme le suggèrent les études épidémiologiques (20). L'apparition d'un changement structural rénal est précoce au cours de l'obésité avec une hypertrophie glomérulaire, une expansion de la matrice mésangiale, un épaississement de la membrane basale glomérulaire et tubulaire et une glomérulo-sclérose focale et segmentaire qui précède les anomalies biologiques décelables (21). Les facteurs aggravants sont le diabète, le syndrome métabolique, l'hypoxie chronique, l'hypertension artérielle et le SAOS.

La perte de poids induite par la diététique, l'activité physique ou la chirurgie bariatrique s'accompagne d'une amélioration de l'atteinte rénale, en particulier de la protéinurie (22).

\section{- Complications socio-psychologiques}

L'obésité est source de préjudice, de discrimination sociale et est associée à une détérioration de la qualité de vie. L'« idéal de minceur » peut participer au développement de troubles du comportement alimentaire (restrictions alimentaires puis compulsions) qui favorisent la prise 
de poids. La dépression peut être déterminante dans la prise de poids mais aussi secondaire, et doit être recherchée et traitée $(23,24)$.

\subsection{Impact médico-économique de l'obésité}

Une étude française de 2006, basée sur les données de l'enquête «Santé protection sociale » de 2002 et sur les données des consommations médicales de soins issues de l'échantillon permanent d'assurés sociaux des 3 principaux régimes de sécurité sociale, estimait le coût annuel lié à l'obésité entre 2.1 et 6.2 milliard d'euros, soit environ 1.5 à $4.6 \%$ des dépenses de santé en 2002 (25). Il n'existe pas de nouvelles études économiques en France mais en Allemagne une étude récente chiffre le coût direct de l'obésité à 29 milliard d'euros par an et le coût indirect à 34 milliard d'euros par an (26). Aux Etats Unis le coût annuel lié à l'obésité est estimé à 149 milliard de dollars en 2016 (27).

\subsection{Traitement chirurgical et endoscopique de l'obésité}

\section{- Techniques endoscopiques}

La cœlioscopie a permis de diminuer la morbimortalité postopératoire immédiate de la chirurgie bariatrique. D'autres alternatives sont recherchées afin de limiter les risques opératoires et les coûts de ces procédures. Les données actuelles portant sur les résultats de ces techniques endoscopiques sont soit insuffisantes, soit de faible niveau de preuve.

Parmi les techniques endoscopiques qui se développent, nous pouvons citer :

- Le ballon intra gastrique. Il s'agit d'une technique restrictive qui vise à induire une satiété précoce et à diminuer l'importance des prises alimentaires. Celui-ci peut être laissé en place pour une durée maximale de six mois et le retrait s'effectue par voie endoscopique. L'efficacité de cette procédure est néanmoins modeste avec une morbidité propre à cette technique (28), (Annexe 1).

- La gastroplastie transorale par suture ou agrafage. Ces techniques de plicature endoscopique verticale, initialement développées pour la prise en charge du reflux gastro-œsophagien, ont été étendues à la prise en charge de l'obésité afin de réaliser par voie endoscopique un manchon gastrique $(29,30)$, (Annexe 2). 
- Les implants endoscopiques restrictif et malabsorptif. Le TERIS ${ }^{\circledR}$ est un dispositif de restriction gastrique combinant la mise en place d'un implant et la plicature endoscopique (31). Il existe deux types d'implants malabsorptifs: le «duodéno-jéjunal bypass sleeve » $\left(\right.$ EndoBarrier $\left.^{\circledR}\right)$ et le « gastro-duodéno-jéjunal bypass sleeve » (ValenTx). Ces deux dispositifs correspondent à un tube fin et flexible fixé au pylore (EndoBarrier ${ }^{\circledR}{ }^{[97]}$ ) ou au cardia (ValenTx ${ }^{[98]}$ ) grâce à un dispositif d'ancrage. La gaine est ensuite déployée dans le duodénum et déroulée jusque dans le jéjunum proximal sous contrôle scopique (EndoBarrier ${ }^{\circledR}$ ) et cœlioscopique (ValenTx). Cette gaine auto expansive forme une barrière entre les aliments et la paroi jéjunale et les sécrétions biliopancréatiques, mimant l'effet d'un bypass à l'origine d'une malabsorption (32). Plusieurs complications majeures motivant l'ablation précoce du dispositif ont été rapportées concernant l'EndoBarrier : occlusion de la gaine (3\%), migration $(13 \%)$, hémorragie digestive haute $(4,3 \%)$ et douleur abdominale $(8,6 \%)$, (Annexe 3$)$.

\section{- La chirurgie bariatrique}

La prise en charge médicale de l'obésité, bien qu'elle soit pluridisciplinaire associant le suivi nutritionnel et psychologique, est peu efficace, avec une perte de poids trop souvent insuffisante et transitoire $(33,(34,35)$.

La chirurgie bariatrique est maintenant reconnue comme le traitement le plus efficace des patients souffrant d'obésité morbide, en terme de perte de poids (36), et d'amélioration des complications de l'obésité (37). A long terme, la chirurgie bariatrique a fourni les preuves d'une réduction de la mortalité (38), ainsi que de la diminution du risque de développer de nouvelles comorbidités, avec une diminution des coûts liés à la santé et à la médicalisation $(39,40)$.

Les différentes interventions chirurgicales sur le tube digestif ont permis une meilleure compréhension des mécanismes impliqués dans les changements métaboliques. Il existe deux grandes catégories de procédures chirurgicales :

- Les techniques fondées sur une restriction gastrique, qui diminuent l'ingestion alimentaire par réduction de la capacité gastrique comme l'anneau gastrique (AG) et la sleeve gastrectomy (SG).

- Les techniques mixtes, qui associent à une restriction gastrique le principe de malabsorption intestinale par la création d'un système de court-circuit ou de dérivation. La 
principale technique est le by-pass, qui est la technique considérée comme apportant les meilleures chances de rémission du diabète de type 2 dans la population de diabète de type 2 présentant une obésité massive (41).

\section{- La sleeve gastrectomy}

La sleeve gastrectomy (SG) est une technique réalisée le plus souvent sous cœlioscopie. Elle consiste en l'exérèse de la grande courbure gastrique, débutant $6 \mathrm{~cm}$ en amont du pylore jusqu'à l'angle de His après calibration du manchon gastrique par un tube de 34 French. Le renforcement de la ligne d'agrafe n'est pas systématique. Un test au bleu de méthylène est réalisé en fin de procédure, après clampage pré pylorique, afin d'éliminer toute fuite de produit sur la ligne d'agrafage (42).

Le mécanisme d'amaigrissement repose alors sur son action restrictive avec une diminution globale du volume gastrique ainsi qu'hormonale avec la diminution de la sécrétion de ghréline qui est une hormone oréxigène produite par l'estomac $(43,44)$.

Cette technique reste efficace, même après $A G$, même si elle expose à un risque plus important de fistule (45).

Ses avantages sont l'absence d'anastomose et de composante malabsorptive, la simplicité opératoire avec une diminution du temps opératoire, de faibles pertes sanguines, une gestion simple de la douleur post-opératoire permettant la diminution des complications médicale (46). Elle permet une perte de poids plus importante que l'AG, et le traitement des comorbidités telles que le SAOS, le diabète de type 2 et des maladies cardiovasculaires (47, 48).

La mortalité de cette chirurgie est de l'ordre de $0.20 \%$ ou $0.3 \%$ (49), et les complications précoces sont l'hémorragie digestive, de l'ordre de $1 \%-1.5 \%(50,51)$, la fistule gastrique (FG), dont l'incidence est estimée à $2.5 \%$ (52), et la sténose, de l'ordre de 0.8 à $3.5 \%$ (53, $54)$.

\section{- La fistule gastrique post sleeve gastrectomy}

L'incidence de la FG post SG est estimée entre $0.7 \%$ et $5 \%$ pour une moyenne de $2.5 \%$ (55, $56,57,52$ ). Dans la plupart des cas, celle-ci survient à la partie haute de la ligne d'agrafes. Elle est définie par une fuite de contenu luminal au niveau d'une anastomose chirurgicale entre 2 organes creux (58). 
Les signes cliniques évocateurs sont la tachycardie, les signes de sepsis, les douleurs abdominales généralement localisées dans l'hypochondre gauche et irradiant à l'épaule gauche. La FG post SG peut parfois être pauci symptomatique et le diagnostic de fistule se révèle sur un examen d'imagerie mettant en évidence une extravasation de produit de contraste hors de la lumière gastrique ou une collection hydro-aérique sous diaphragmatique dans le quadrant abdominal supérieur gauche. La présentation clinique est donc variable et peut se présenter aussi bien de manière fortuite que par un tableau de péritonite avec choc septique et défaillance multi viscérale.

Les hypothèses physiopathologiques expliquant la survenue d'une FG post SG sont une ischémie tissulaire induite par la suture opératoire et des causes mécaniques telles qu'une tension ou une suture incomplète. Dans les deux cas, la pression intraluminale dépasse la résistance du tissu gastrique et de la ligne d'agrafes, entrainant l'apparition d'une fuite. Il est généralement admis que les fistules précoces apparaissant dès $48 \mathrm{~h}$ sont en général de cause mécanique tandis que les fistules plus tardives sont liées à une cause ischémique par tension de la suture, une occlusion digestive en aval ou par la présence d'un hématome $(59,60)$.

Actuellement, il n'existe aucun consensus sur la prise en charge médicale, radiologique et chirurgicale des FG post SG. Après avoir confirmé la présence de la FG, une mise à jeun orale stricte est initiée, associée à un support nutritionnel par voie entérale ou parentérale. Le drainage de la collection est réalisé par voie chirurgicale ou radiologique, sous couvert d'antibiotiques parentéraux. Le choix de l'abord, chirurgical ou radiologique, dépend de l'état septique et hémodynamique du patient, de l'accessibilité radiologique des collections et des compétences de chaque centre.

Différentes techniques complémentaires ont été décrites pour le traitement des FG post SG dont le principe est de couvrir ou fermer la fistule afin de favoriser la guérison.

La technique endoscopique la plus décrite repose sur l'utilisation de prothèse métallique couverte œsophagienne (SEMS). L'objectif de cette technique est de couvrir la fistule par une prothèse métallique extirpable sous contrôle fluoroscopique afin de favoriser la cicatrisation. Le taux de guérison est inconstant, variant de $19 \%$ à $95 \%$ selon des études de faible niveau de preuve (cohortes rétrospectives de faibles effectifs) $(61,62,63)$.

La principale complication principale est la migration de la prothèse variant de $5 \%$ à $62 \%$ selon les études $(61,64)$, et nécessitant la mise en place de nouvelles prothèses 
endoscopiques. Les prothèses métalliques partiellement couvertes seraient supérieures aux prothèses couvertes dans la prévention de la migration (65). Néanmoins, les SEMS sont souvent mal tolérées, occasionnant des douleurs, un reflux gastro-œsophagien ou des vomissements, pouvant motiver l'ablation de celles-ci. La complication la plus grave est la survenue d'hémorragie digestive sur pathologie ulcéreuse où de rares cas de fistules avec l'aorte ont été décrites $(66,67)$.

Une autre stratégie endoscopique consiste, à obturer la fistule. L'utilisation de colle biologique au sein de FG post SG n'a pas montré une réelle efficacité (68). Une méthode prometteuse utilise des clips OTSC de type OVESCO®, dont le succès clinique varie de 42 à $91 \%(69,70,71)$.

Les effectifs des différentes études sont faibles et l'usage de clips OTSC est souvent préconisé en complément des prothèses œsophagiennes afin de limiter la migration de cellesci ou en cas de fistule de petit calibre $(72,73,74)$.

Une nouvelle approche développée au CHU d'Amiens et dénommée la technique Bartoli, consiste à effectuer un drainage interne des collections péri-orificielles à l'aide de prothèses double queue de cochon au travers de l'orifice fistuleux et de diriger la fermeture de la FG de l'extérieur vers l'intérieur. Ce processus semblant plus physiologique permet de rétablir le flux vers la lumière digestive du fait d'un gradient de pression favorable et d'obtenir une fermeture rapide de l'orifice de drainage externe. Cette technique est inspirée de la technique de gastrostomie endoscopique utilisée dans le traitement des pseudo-kystes pancréatiques (75). Cette prise en charge n'est pas dépendante du délai de survenue de la fistule, mais de la présentation clinique et radiologique. Une évaluation préliminaire portant sur 47 patients traités par prothèses double queue de cochon (PDQC) retrouvait un taux de guérison de $91,6 \%$ et une durée médiane de prise en charge de 80 jours. Le délai optimal pour la réalisation d'une chirurgie radicale avec anastomose fistulo-jéjunale, en cas d'échec du traitement endoscopique, était de 120 jours (76).

\subsection{Objectif de l'étude}

L'objectif principal de l'étude était d'évaluer l'efficacité des PDQC selon la technique Bartoli dans le traitement des FG après SG. 


\section{PATIENTS ET METHODES}

\subsection{Patients}

\section{- Critères d'inclusion}

Entre janvier 2010 et avril 2016 (PT), tous les patients ayant une FG après SG et ayant bénéficié de la pose de PDQC lors de la première endoscopie réalisée au CHU d'Amiens ont été inclus consécutivement et analysés rétrospectivement.

\section{- Critères d'exclusion}

Les patients n'étaient pas inclus dans l'étude en cas de présence d'une FG post chirurgie bariatrique autre que la sleeve gastrectomy, de fistule localisée à un autre site que le pôle supérieur de la ligne d'agrafes, d'une fistulisation à l'étage sus diaphragmatique ou gastrocutanée. Les patients ayant bénéficié d'une autre technique endoscopique lors de la première endoscopie étaient aussi exclus.

\subsection{Recueil des données}

- Méthodes

Les patients ont été inclus consécutivement et prospectivement dans une base de données et analysés rétrospectivement. Les données ont été saisies sur un tableur Excel. Les inclusions se sont déroulées de janvier 2010 à avril 2016 sur une durée totale de 75 mois.

La récupération des données a été effectuée à l'aide à partir des dossiers médicaux par appel téléphonique aux différents services de chirurgie digestive des hôpitaux ou cliniques initiaux (Centres hospitaliers de Laon, Compiègne, Creil, Beauvais, Boulogne sur Mer, Dieppe, Abbeville, Charleville Mézières, Cambrai, Saint-Quentin, Senlis, Léman et Fourmies) et par appel téléphonique aux patients et médecins traitants.

\section{- Variables recueillies}

Les caractéristiques des patients recueillies étaient l'âge, le poids, le sexe, l'IMC avant opération et les comorbidités.

Les données liées à la chirurgie bariatrique comprenaient la date de la chirurgie, le centre initial, la notion de chirurgie bariatrique antérieure (AG ou SG) et les modalités de la SG. 
Les données liées au diagnostic de la fistule comprenaient la date, le délai par rapport à la chirurgie, la taille de la fistule estimée par l'endoscopiste et les paramètres biologiques au diagnostic (bilan inflammatoire et nutritionnel). Les données liées à la prise en charge initiale de la FG comprenaient en cas de nécessité d'un drainage externe, les modalités du drainage radiologique ou chirurgical, notamment le délai d'ablation des modules de drainage par rapport à la reprise chirurgicale et à la première endoscopie.

Les données liées au drainage endoscopique comprenaient principalement le taux de drainage endoscopique premier en l'absence de drainage externe, le nombre d'endoscopies thérapeutiques, la présence de complications (migration, hémorragie, perforation) le nombre de pose de PDQC par endoscopie, en cas de migration le taux de recours à une nouvelle pose de PDQC, la présence d'une sténose associée et le taux d'échec.

Les données liées à l'hospitalisation comprenaient la durée d'hospitalisation totale, la durée d'hospitalisation en réanimation, et la durée du traitement.

Les données liées à l'alimentation comprenaient la nécessité de recours à un support nutritionnel parentéral par port-à-cathéter (PAC) ou entéral par sonde naso-jéjunale (SNJ) ou jéjunostomie, ainsi que les paramètres nutritionnels au diagnostic et à la cicatrisation.

\subsection{Procédure de traitement des fistules gastriques post sleeve gastrectomy}

\section{- Prise en charge initiale}

Après confirmation diagnostique, la prise en charge initiale consistait à la mise à jeun orale stricte, un traitement par inhibiteurs de la pompe à protons (IPP), une antibiothérapie probabiliste active sur les bacilles gram négatifs et les germes anaérobies après prélèvements bactériologiques et un support nutritionnel (nutrition parentérale sur PAC ou nutrition entérale sur SNJ ou jéjunostomie d'alimentation), (Annexe 6).

\section{- Indication et modalité du drainage externe}

Le drainage externe et ses modalités (par voie chirurgicale sous anesthésie générale ou par voie radiologique sous anesthésie locale ou générale) étaient indiqués en fonction de la tolérance du sepsis et des données du scanner initial. Une collection bien organisée est définie en tomodensitométrie par une collection bien limitée, encapsulée sans épanchement péritonéal libre. Une instabilité sur le plan septique est définie par l'association d'un syndrome de 
réponse inflammatoire systémique (SRIS) à des critères d'infection bactérienne présumée. Le SRIS nécessite la présence d'au moins 2 des critères suivants (Tableau 1) :

\begin{tabular}{|c|}
\hline Température $>38,3{ }^{\circ} \mathrm{C}$ ou $<36{ }^{\circ} \mathrm{C}$ \\
\hline Fréquence cardiaque $>90 \mathrm{~b} / \mathrm{min}$ \\
\hline Fréquence respiratoire $>20 \mathrm{c} / \mathrm{min}$ \\
\hline Glycémie $>7,7 \mathrm{mmol} / \mathrm{l}$ \\
\hline $\begin{array}{c}\text { Leucocytes }>12000 / \mathrm{mm}^{3} \text { ou }<4000 / \mathrm{mm}^{3} \text { ou }>10 \% \text { de formes } \\
\text { immatures }\end{array}$ \\
\hline
\end{tabular}

Tableau 1 : Critère de SRIS selon International Sepsis Definitions Conference.(Levy MM et al Crit Care Med 2003)

En cas de collection abcédée bien organisée responsable d'une instabilité sur le plan septique, ou de collection mal organisée, un drainage externe est effectué.

Le drainage chirurgical consistait en la réalisation d'une toilette péritonéale avec prélèvements bactériologiques puis au repérage de l'orifice fistuleux, qui pouvait être facilité, après clampage de l'antre, par l'injection, via une sonde naso-gastrique, de bleu de méthylène ou d'air, puis à la mise en place de lames ou de deux drains de Shirley au contact de l'orifice fistuleux. Le premier drain était mis en aspiration douce à moins $50 \mathrm{~cm}$ d'H20 et un lavage par une solution saline (1 à 2 litres) était effectué sur le second. La voie coelioscopique était privilégiée lorsque l'état hémodynamique du patient le permettait. La suture endoscopique était principalement réalisée en cas de fistule précoce survenant dans les 48 premières heures, (Annexe 7).

Le drainage radiologique consistait en la réalisation d'une anesthésie locale ou générale d'une ponction percutanée de la collection sous contrôle échographique ou scannographique, avec la réalisation de prélèvements bactériologique, puis à la mise en place d'un ou plusieurs drains dans la collection après dilatation du trajet percutané. Un lavage adapté à la collection, au nombre et au calibre des drains était effectué.

Une endoscopie était par la suite réalisée pour réaliser un drainage interne de la fistule.

En cas de collection bien organisée chez un patient stable sur le plan septique, aucun drainage externe n'était réalisé. Le drainage endoscopique était alors réalisé dès le diagnostic de fistule posé, (Schéma 1). 


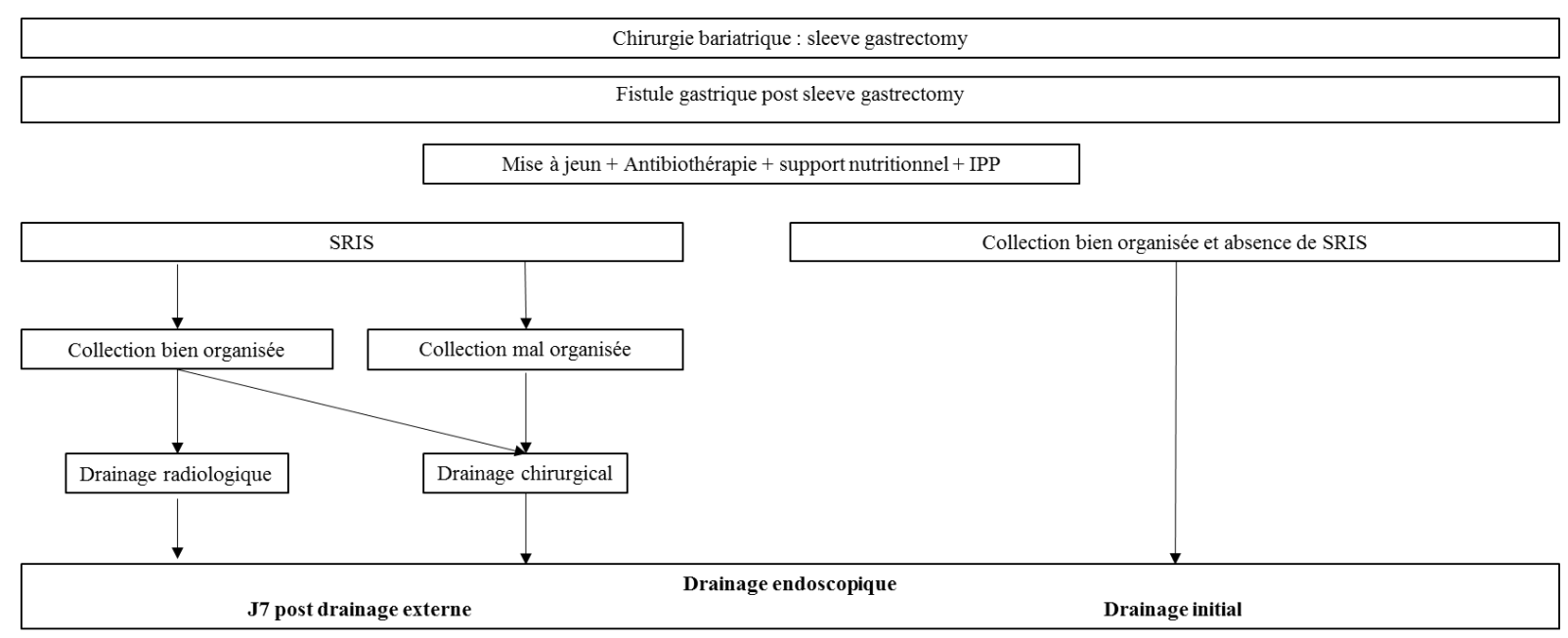

Schéma 1 : Prise en charge de la fistule gastrique post sleeve gastrectomy selon la technique Bartoli

\section{- Modalités du drainage endoscopique}

Le patient bénéficiait sous anesthésie générale d'un drainage interne par PDQC à l'aide d'un fibroscope sous contrôle fluoroscopique. L'utilisation d'une insufflation au CO2 s'est généralisée à partir de septembre 2015. Une visualisation directe de l'orifice primaire, aidée d'une opacification par du produit de contraste, permettait la caractérisation de la fistule (fistule simple ou complexe), sa localisation, son diamètre, et la présence d'une sténose associée, (Annexe 8). La prise en charge endoscopique était assurée par six médecins expérimentés en endoscopie interventionnelle.

La prise en charge des orifices fistuleux consistait en la montée en trans-fistule d'un fil guide (JagWire straight tip 0.035 in $\times 450 \mathrm{~cm}$ ou DreamWire straight tip 0.035 in $\times 450 \mathrm{~cm}$; Boston scientific, Marlborough, Massachussetts, USA) aidé par un cathéter (cannula standard Tip $210 \mathrm{~cm} \times 5$ Fr; Boston Scientific, Marlborough, Massachussetts, USA.). Le cas échéant, une mobilisation per-endoscopique des modules de drainage externe était réalisée pour laisser un espace de $2-3 \mathrm{~cm}$ entre les modules de drainage externe et les PDQC. Deux PDQC de 7 French étaient mises en place pour permettre un drainage intra-gastrique de la collection. La longueur de la prothèse dépendait des constatations endoscopiques, mais dans la grande majorité des cas, la longueur estimée était de $5 \mathrm{~cm}$ (Zimmon biliary stent set, 7Fr, $5 \mathrm{~cm}$; Cook médical, Bloomington, Indiana, USA), (Annexe 9 à 12). La pose d'un drain naso-fistule (single use nasal biliary drainage tube V, Quick Place V, $2550 \mathrm{~mm} \times 7 \mathrm{Fr}$; Olympus, Shinjuku, Tokyo, Japon) était réalisée en cas d'issue de pus franc afin de laver la cavité. 
La prise en charge de sténose associée consistait en une dilatation au ballon pneumatique. L'efficacité était démontrée par un passage de l'endoscope sans difficulté et par un passage correct du produit de contraste en fluoroscopie. En l'absence de jéjunostomie, une sonde naso-jéjunale était posée en per endoscopie (kangaroo, naso jejunal feeding tube $7 \mathrm{~g}$ weighted tip $12 \mathrm{Fr} \times 152 \mathrm{~cm}$; Covidien, Dublin, Irlande)

En post interventionnel, les modules de drainages externes étaient progressivement retirés pour permettre un drainage interne de la collection via les prothèses double queue de cochon.

En l'absence de signes d'infection à l'ablation des modules de drainage externe, le patient était autorisé à retourner au domicile.

Plusieurs modalités de gestion du drainage ont été évaluées au cours de la période janvier 2010 - octobre 2013 (P1). Les modalités du drainage chirurgical évaluées portaient notamment sur l'utilisation de lames ou de Shirley ainsi que sur leur nombre et leur durée en post endoscopie. Les modalités du support nutritionnel ont évolué avec l'utilisation prépondérante d'une nutrition entérale par jéjunostomie ou sonde naso-jéjunale au détriment de la nutrition parentérale. L'évolution du traitement endoscopique s'est portée sur une restriction du nombre d'endoscopies (diagnostiques ou thérapeutiques), sur un raccourcissement du délai de réévaluation tomodensitométrique et endoscopique, sur le nombre et les caractéristiques des PDQC et sur le développement de techniques dérivées pour le traitement des larges fistules utilisant de manière combinée un SEMS associé à un drainage par des PDQC de la fistule (traitement combiné), (Annexe 13). Cette évolution des modalités de drainage pendant la période $\mathrm{P} 1$ a permis de codifier le recours à une chirurgie radicale en cas d'échec de la technique et donc d'aboutir à une standardisation de la technique Bartoli lors de la période $\mathrm{P} 2$ de novembre 2013 à avril 2016 (P2).

Cette standardisation repose sur :

- La réalisation d'un drainage radiologique en cas de collection bien collectée chez un patient mal contrôlé sur le plan septique.

- La réalisation d'une toilette chirurgicale avec utilisation quasi exclusive de drains de Shirley, au nombre de deux, en cas de collection mal organisée ou de sepsis incontrôlé.

- La réalisation d'un drainage interne initial par endoscopie par PDQC en cas de collection bien organisée chez un patient contrôlé sur le plan septique. 
- Un support nutritionnel entéral privilégié.

- La généralisation de l'utilisation du CO2.

- La réalisation d'une endoscopie systématique pour drainage interne sept jours après la réalisation d'un drainage externe efficace.

- La pose de deux PDQC au minimum, mesurant 7 French et $5 \mathrm{~cm}$ en cas de fistule de petite taille et l'utilisation d'un traitement combiné par SEMS associé à la pose PQDC en trans-SEMS en cas de fistule large ou de sténose associée.

- Une réévaluation tomodensitométrique et endoscopique entre quatre et six semaines après la réalisation du drainage interne.

- Le recours à une chirurgie par anastomose gastro-jéjunale au contact de la fistule après échec du traitement endoscopique, notamment après dix-huit semaines d'évolution.

\subsection{Evaluation au cours du suivi}

\section{- Critère de jugement principal}

Le critère de jugement principal était l'efficacité sur la cicatrisation du drainage interne par PDQC selon la technique Bartoli dans le traitement des FG après SG pour les périodes PT, P1 et P2. L'efficacité de la technique était définie par la cicatrisation de la FG permettant une réalimentation orale en l'absence de collection abdominale, de sepsis, de prothèse endoscopique et de module de drainage externe. La période PT correspondait à la période totale d'inclusion, soit de janvier 2010 à avril 2016, la période P1 correspondait à la période de janvier 2010 à octobre 2013 et la période P2 correspondait à la période de novembre 2013 à avril 2016.

\section{- Critères de jugement secondaire}

Les critères de jugement secondaires étaient :

- Le succès technique défini par la pose endoscopique de PDQC dans l'orifice fistuleux.

- L'efficacité de tous les traitements endoscopiques définie par une efficacité sur la cicatrisation des FG par l'emploi éventuellement combiné des prothèses métalliques (SEMS) couvertes.

- Le délai médian de guérison de la fistule défini par le délai entre la première endoscopie et la cicatrisation de la FG. 
- Le taux médian d'endoscopie première pour drainage interne ainsi que le taux médian de recours à un drainage chirurgical ou radiologique et la durée médiane de drainage externe par rapport à la première endoscopie.

- Le nombre médian d'endoscopies thérapeutiques définies par la réalisation d'un geste à visée thérapeutique (pose ou ablation de PDQC ou d'un drain naso fistule) et le nombre moyen d'endoscopies avec mise en place de PDQC par patient.

- Le taux médian de complications liées à la technique Bartoli.

- La tolérance clinique définie par l'apparition de symptômes cliniques ou biologiques liés à la pose de PDQC.

- La durée médiane d'hospitalisation totale, le taux médian et la durée médiane d'hospitalisation en unité de réanimation.

- Le taux médian de chirurgie radicale (anastomose gastro-jéjunale au contact de la FG)

- La mortalité.

\subsection{Analyse statistique}

Les variables quantitatives ont été décrites à l'aide de la médiane (premier et troisième quartile) ou de la moyenne (extrêmes) et les variables qualitatives ont été décrites en nombre (pourcentages). La comparaison des variables quantitatives a été réalisée par le test t de Student. Pour les variables qualitatives, un test de chi2 avec correction de Yates a été utilisé. L'analyse univariée a été réalisée par un modèle de régression logistique avec sélection ascendante avec p inférieure ou égale à 0,2 . 


\section{RESULTATS}

\subsection{Caractéristiques des patients}

L'étude s'est déroulée de janvier 2010 à avril 2016 au Centre Hospitalier d'Amiens Picardie. Quatre-vingt-deux patients ont été pris en charge pendant la période PT, dont 34 pour la période $\mathrm{P} 1$ et 48 pour la période $\mathrm{P} 2$.

Les caractéristiques des patients sont représentées dans le tableau 1.

Pour PT, l'âge médian était de 33.5 ans (IQR 28.25-44.75) avec une prédominance féminine qui représente $76.8 \%$ de l'effectif. Les patients présentaient une obésité morbide avec un IMC médian à 42.7 kg/m2 (IQR 39.85-43.37). La prévalence de l'HTA était de $17.1 \%$, du diabète de $15.8 \%$, des dyslipidémies de $14.6 \%$ et du SAOS de $28 \%$. Il n'existait pas de différence entre les populations des deux périodes $\mathrm{P} 1$ et $\mathrm{P} 2$.

Sur le plan chirurgical, $56.1 \%$ des patients pris en charge dans notre centre étaient référés par d'autres hôpitaux et cliniques ; 15,8\% des patients avaient bénéficié d'une précédente chirurgie bariatrique avant la SG, soit $20.6 \%$ des patients pour la période $\mathrm{P} 1$ et $12.5 \%$ pour la période P2 ( $\mathrm{p}=\mathrm{NS})$. L'AG était la principale prise en charge bariatrique précédant la SG avec $13.4 \%$ des patients pour la période PT.

Le délai médian du diagnostic de fistule était de 9.5 jours (IQ 4-22), et 1'examen diagnostic principal était dans $79.3 \%$ des cas le scanner abdomino-pelvien avec injection de produit de contraste et opacification haute. La taille de la fistule était connue dans $67 \%$ des cas, celle-ci étant appréciée par l'endoscopiste. La prévalence des fistules de petite et grande taille était respectivement de $39 \%$ et $28 \%$. La prévalence des sténoses associées était de $3.7 \%$. 


\begin{tabular}{|c|c|c|c|c|}
\hline & PT & $\mathrm{P} 1$ & $\mathrm{P} 2$ & $\mathrm{p}$ \\
\hline Nombre de patient & $\mathrm{n}=82$ & $n=34$ & $\mathrm{n}=48$ & \\
\hline $\begin{array}{l}\text { Sexe : } \\
\text { Homme (n, \%) }\end{array}$ & $19(23.2)$ & 7 (20.6) & $12(25)$ & \multirow{2}{*}{ NS } \\
\hline Femme $(\mathrm{n}, \%)$ & $63(76.8)$ & $27(79.4)$ & $36(75)$ & \\
\hline Age médian (années, IQR) & $\begin{array}{l}33.5(28.25- \\
\quad 44-75)\end{array}$ & $\begin{array}{c}36.5(29- \\
46.75)\end{array}$ & $32.5(28-40)$ & NS \\
\hline Poids médian kg (IQR) & $\begin{array}{c}120 \\
(108.2-133.5)\end{array}$ & $\begin{array}{c}122 \\
(115.25- \\
135.5)\end{array}$ & $\begin{array}{c}117.5 \\
(107.25- \\
130.25)\end{array}$ & NS \\
\hline IMC médian $(\mathrm{kg} / \mathrm{m} 2)$ & $\begin{array}{l}42.7(39.85- \\
43.37)\end{array}$ & $\begin{array}{c}43.2(40- \\
48.92)\end{array}$ & $\begin{array}{l}41.9(39.5- \\
45.07)\end{array}$ & NS \\
\hline HTA (n, \%) & $14(17.1)$ & $5(14.7)$ & $9(18.7)$ & NS \\
\hline Dyslipidémie (n, \%) & $2(14.6)$ & 7 (20.6) & $5(10.4)$ & NS \\
\hline Diabète (n, \%) & $13(13.8)$ & 7 (20.6) & $6(12.5)$ & NS \\
\hline SAOS (n, \%) & $23(28)$ & $8(23.5)$ & $15(31.2)$ & NS \\
\hline Syndrome métabolique (n, \%) & $4(4.9)$ & $2(5.9)$ & $2(4.2)$ & NS \\
\hline $\begin{array}{l}\text { Centre chirurgical initial : CHU } \\
\text { Amiens (n, \%) }\end{array}$ & $36(43.9)$ & $18(52.9)$ & $18(37.5)$ & NS \\
\hline $\begin{array}{l}\text { Centre chirurgicale initial : centre } \\
\text { hospitalier et clinique extérieure } \\
(\mathrm{n}, \%)\end{array}$ & $46(56.1)$ & $16(47.1)$ & $30(62.5)$ & NS \\
\hline $\begin{array}{l}\text { Chirurgie bariatrique antérieure } \\
(\mathrm{n}, \%) \text { : }\end{array}$ & $13(15.8)$ & 7 (20.6) & $6(12.5)$ & \\
\hline $\mathrm{AG}$ & $11(13.4)$ & $5(14.7)$ & $6(12.5)$ & NS \\
\hline SG & $1(1.2)$ & $1(2.9)$ & 0 & \\
\hline $\mathrm{SG}+\mathrm{AG}$ & $1(1.2)$ & $1(2.9)$ & 0 & \\
\hline $\begin{array}{l}\text { SG }(\mathrm{n}, \%): \\
\text { par cœlioscopie }\end{array}$ & $75(91.5)$ & $28(82.3)$ & 47 (97.9) & \\
\hline Par laparoscopie & $5(6.1)$ & $4(11.8)$ & $1(2.1)$ & NS \\
\hline $\mathrm{NC}$ & $2(2.4)$ & $2(5.9)$ & 0 & \\
\hline $\begin{array}{l}\text { Délai diagnostic médian de la FG } \\
\text { (jours) }\end{array}$ & 9.5 & 9 & 10 & NS \\
\hline Taille fistule $(\mathrm{n}, \%)$ : & & & & \\
\hline Non connue & $27(33)$ & $11(32.4)$ & $16(33.3)$ & \\
\hline Petite & $32(39)$ & $13(38.2)$ & $19(39.6)$ & NS \\
\hline Grande & $23(28)$ & $10(29.4)$ & $13(27.1)$ & \\
\hline Sténose associée (n, \%) & $3(3.6)$ & $1(2.9)$ & $2(4.2)$ & NS \\
\hline
\end{tabular}

Tableau 1 : Caractéristiques des patients pour PT, P1 et P2.

PT : période totale de janvier 2010 à avril 2016, P1 : période 1 de janvier 2010 à octobre 2013, P2 : période 2 de novembre 2013 à avril 2016, IMC : indice de masse corporelle (kg/m2), HTA : hypertension artérielle, SAOS : syndrome d'apnée obstructive du sommeil, CHU : centre hospitalo-universitaire, SG : sleeve gastrectomy, AG : anneau gastrique, FG : fistule gastrique. 


\subsection{Efficacité de la technique Bartoli}

L'efficacité de la technique Bartoli sur la cicatrisation des FG liées à la SG était de 79.2\% pour PT (n=65), $70.5 \%$ pour P1 $(n=24)$ et de $85.4 \%$ pour P2 $(n=41) ;(p=0.02)$ (Figure 1$)$.

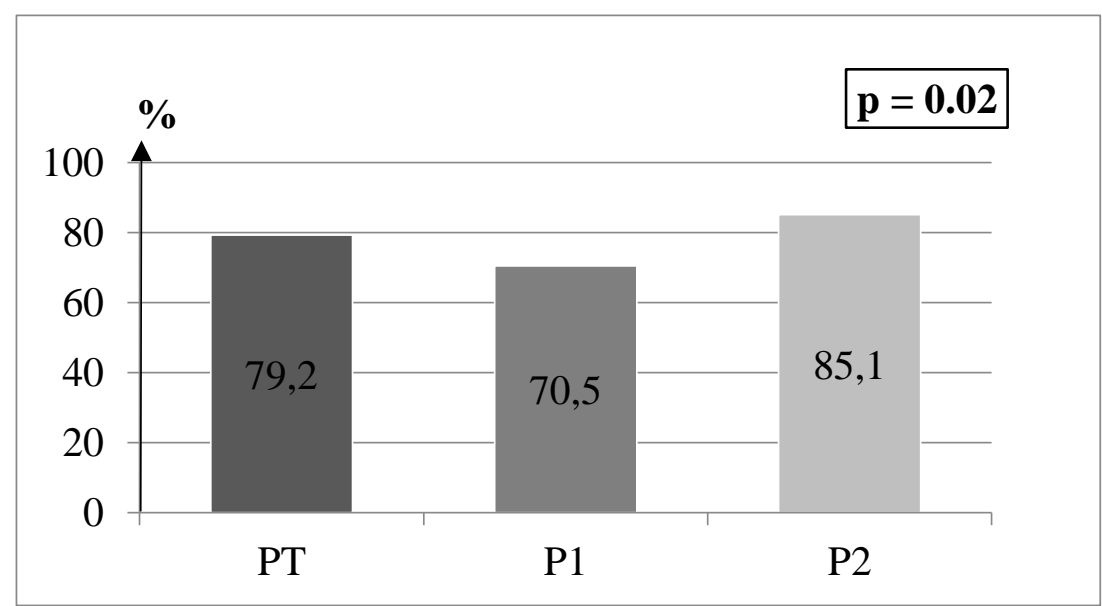

Figure 1 : Taux d'efficacité de la technique Bartoli pour la période totale (PT : janvier 2010 à avril 2016), P1 (janvier 2010 - octobre 2013) et P2 (novembre 2013-avril 2016).

\subsection{Efficacité des traitements endoscopiques}

L'efficacité de l'ensemble des traitements endoscopiques était de 97.6\% $(\mathrm{n}=80)$ pour la période PT. Parmi les 17 patients en échec primaire de la technique Bartoli, 11\% (n=9) étaient traités efficacement par la pose de SEMS et 7.3\% $(n=6)$ par un traitement combiné utilisant des PDQC posées au travers de SEMS (Figure 2).

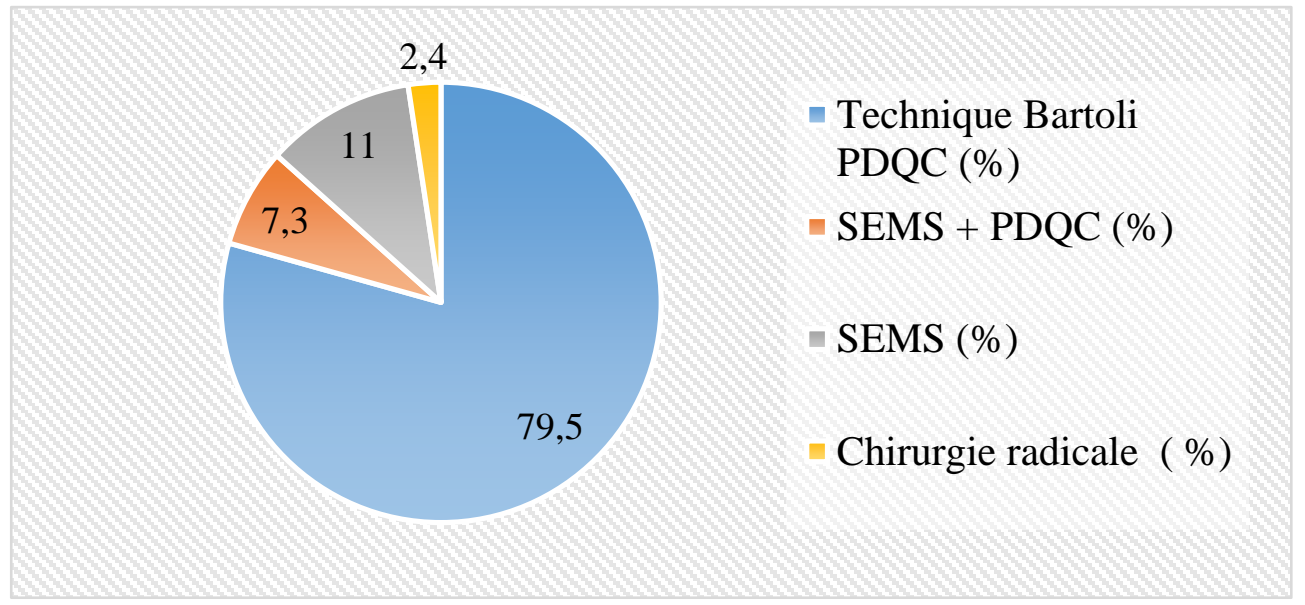

Figure 2 Efficacité thérapeutique des différents traitements des FG post SG pour la période PT (\%). 


\subsection{Evaluation des modalités de la technique Bartoli pour la période PT}

\subsubsection{Evaluation des modalités du drainage externe}

Un drainage endoscopique premier a pu être réalisé dans $36.6 \%(n=30)$ alors qu'un drainage externe initial a été nécessaire pour $63.4 \%(\mathrm{n}=52)$ des patients; $12.2 \%(\mathrm{n}=10)$ par abord radiologique et $51.2 \%(n=42)$ par abord chirurgical.

Le cas échéant, la première endoscopie était réalisée dans un délai médian de 8 jours (IQR 612.5) après la réalisation du drainage radiologique ou chirurgical. La durée médiane du drainage externe après la première endoscopie était de 4 jours (IQR 3-11) pour le drainage chirurgical et de 7 jours (IQR 3.5-9.75) pour le drainage radiologique. Onze pourcents des patients $(n=9)$ ont bénéficié d'une reprise chirurgicale $(n=4)$ ou radiologique post endoscopie $(\mathrm{n}=5)$, dont l'indication principale était un drainage externe insuffisant avec persistance d'une collection ou d'un SRIS $(n=6)$ ou nécessité d'un nouveau drainage après perforation lors de la pose de PDQC (n=3) (Figure 3).

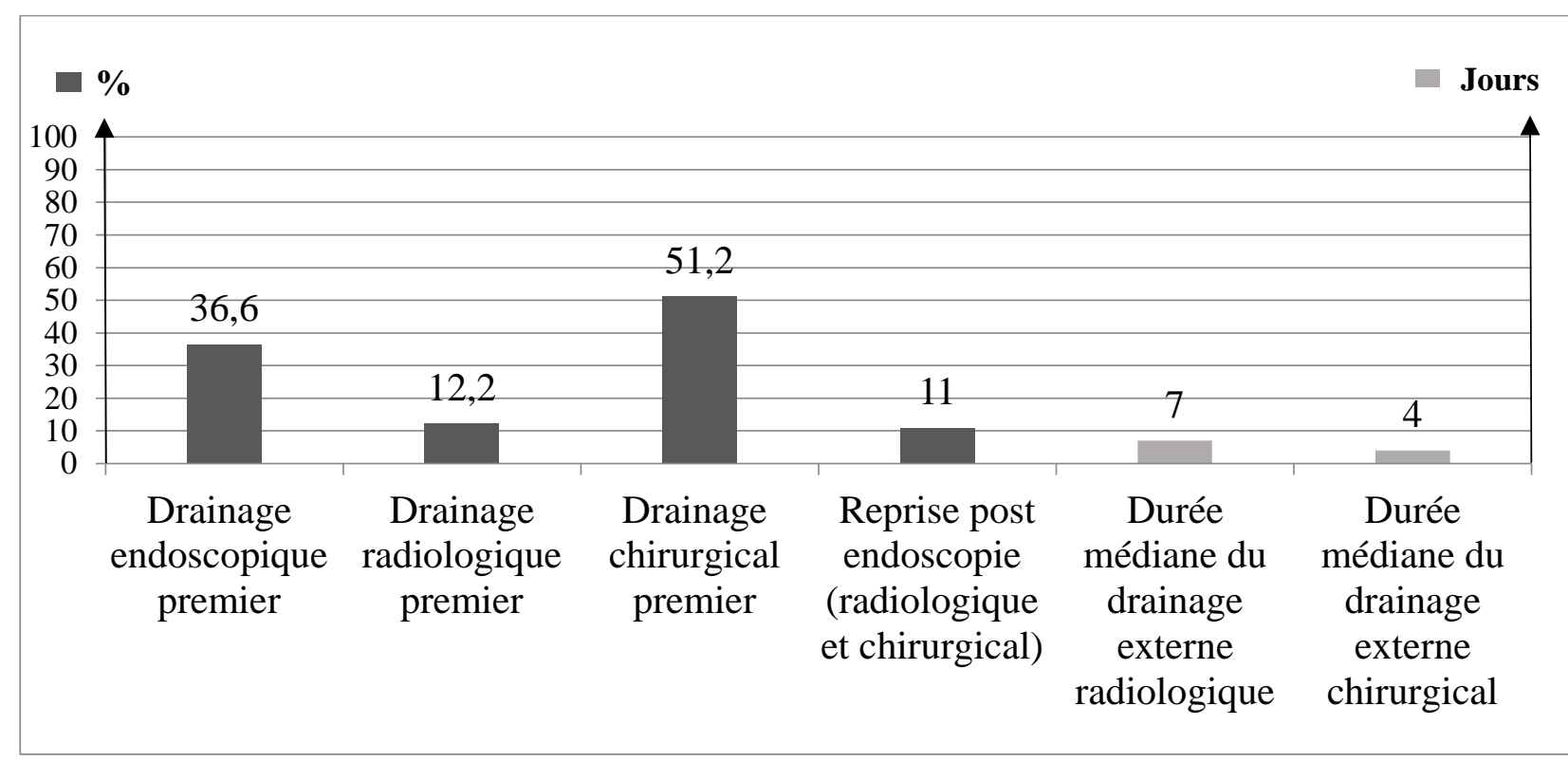

Figure 3 : Evaluation des modalités du drainage externe et du taux de drainage endoscopique premier pour la période PT 


\subsubsection{Evaluation de la tolérance et des complications}

La tolérance était à 97.6\%. Deux patients rapportaient des douleurs retro-sternales et nausées après la pose de PDQC s'amendant rapidement sous traitement symptomatique et ne nécessitant pas l'ablation des prothèses

Le taux de migration digestive était de $19.5 \%(\mathrm{n}=16)$ mais seule la moitié des patients $(\mathrm{n}=8$ soit $9.7 \%$ ) a présenté une migration symptomatique et a nécessité une nouvelle pose de PDQC. Aucune complication hémorragique n'a été observée. Le taux de perforations était de $3.6 \%(n=3)$ et une reprise chirurgicale a été nécessaire pour la réalisation d'une toilette péritonéale avec drainage externe. Parmi les trois perforations, deux patients ont bénéficié d'un second traitement endoscopique de la FG selon la technique Bartoli (Figure 4).

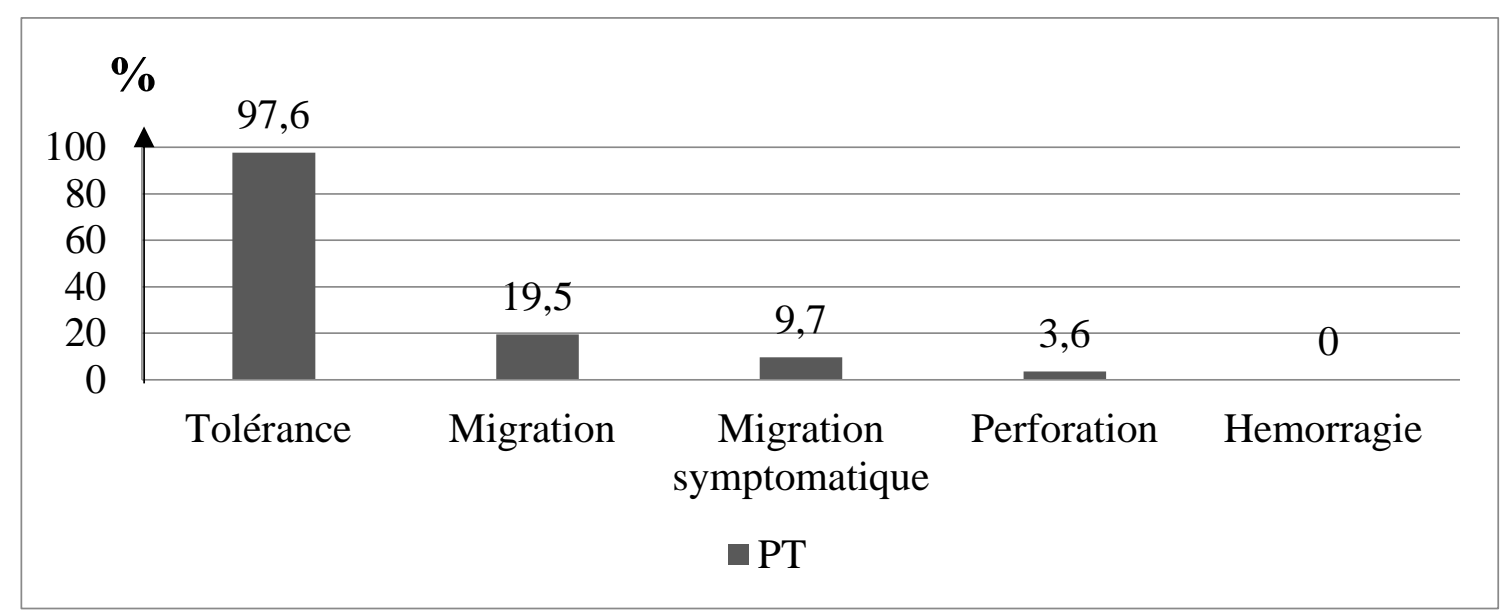

Figure 4 : Evaluation de la tolérance et des complications pour la période PT

\subsubsection{Evaluation des modalités endoscopiques, d'alimentation, d'hospitalisation et de guérison}

Le support nutritionnel préférentiel était la voie entérale ; $50 \%$ des patients bénéficiaient d'une jéjunostomie d'alimentation, $37.8 \%$ d'une SNJ et $6.1 \%$ d'un support parentéral via la mise en place d'un port-à-cathéter (PAC).

Le nombre médian et la durée totale médiane d'hospitalisation étaient respectivement de 2 (IQR 2-3) et de 18.5 jours d'hospitalisation (IQR 13-31). Le recours à une unité de réanimation ou de soins intensifs était nécessaire pour $34.1 \%(\mathrm{n}=28)$ des patients avec une durée médiane de séjour de 15 jours (IQR 5.5-21), (Figure 5). 
Le succès technique était de 98,8\% ( $\mathrm{n}=81)$. Une perforation per endoscopique a nécessité une prise en charge chirurgicale et n'a pas autorisé la mise en place ultérieure de PDQC.

Le nombre médian d'endoscopies thérapeutiques était de 2 (IQR 2-3.75). Le nombre moyen par patient d'endoscopies avec pose de PDQC était de 1.6 (extrêmes 1-7). Il n'y a eu aucun décès constaté.

Deux patients $(2,4 \%)$ ont bénéficié d'une chirurgie radicale par anastomose gastro-jéjunale au contact de la FG. Le délai médian de guérison de la fistule était de 58 jours (IQR 46-76), (Figure 5).

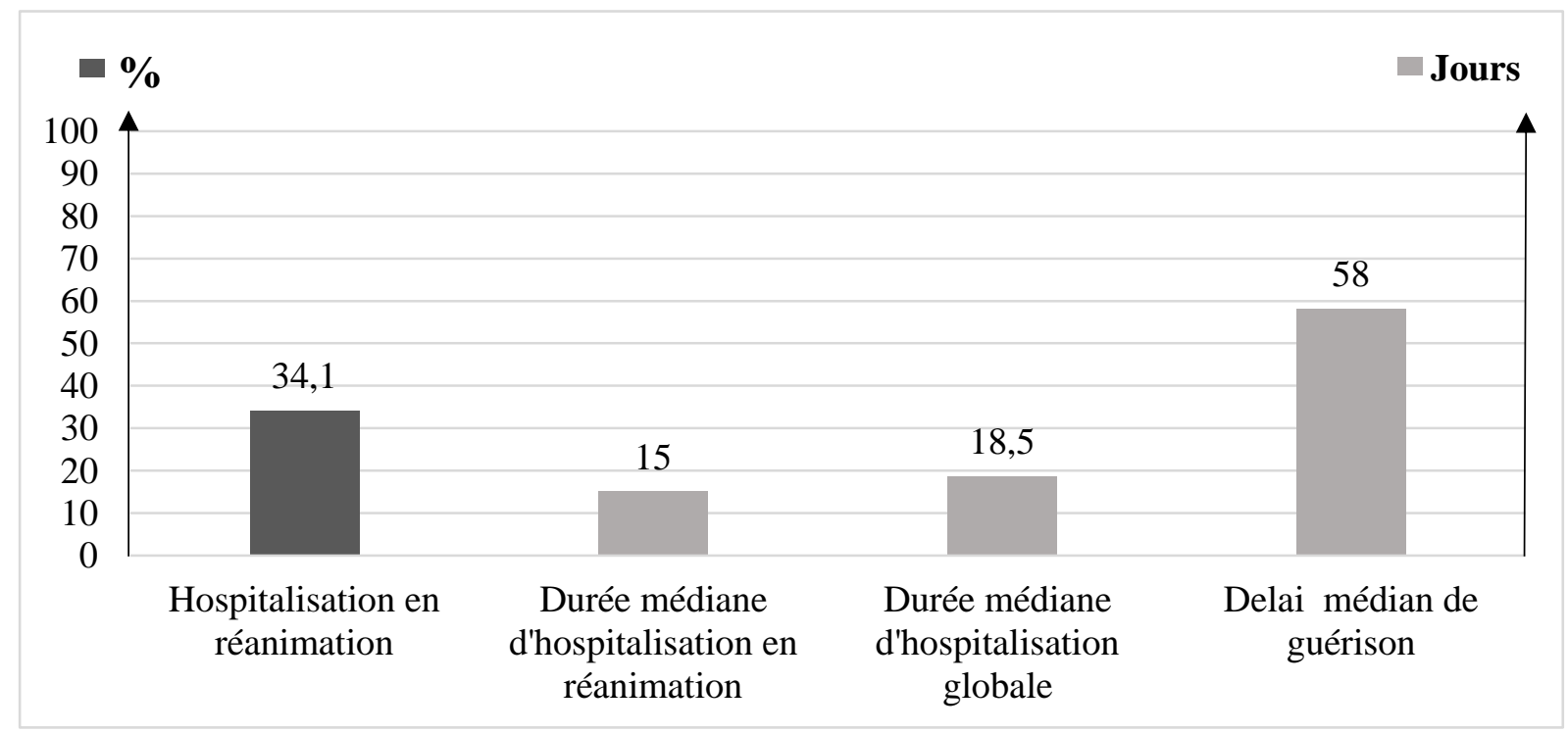

Figure 5 : Modalités d'hospitalisation et de guérison pour la période PT

\subsection{Evaluation de la technique Bartoli pour les périodes P1 et P2, (Tableau 3)}

L'efficacité de la technique Bartoli sur la cicatrisation des FG liée à la SG était de $85.4 \%$ pour $\mathrm{P} 2$ et de $70.5 \%$ pour P1 $(\mathrm{p}=0.02)$. L'efficacité du traitement endoscopique associant la technique Bartoli et le traitement combiné (SEMS associé aux PDQC) était de $93.7 \%$ et de $76.4 \%$ respectivement pour P2 et P1 ( $\mathrm{p}=0.02)$, (Figure 6). Le délai de guérison était de de 48 jours pour P2 (IQR 42.25-69) et 70.5 jours pour P1 (IQR 57.5-85) $(\mathrm{p}=0.0017)$. 
Un drainage endoscopique a été réalisé en première intention pour $41.7 \%$ des patients pour $\mathrm{P} 2$ et $29.4 \%$ des patients pour P1. La durée médiane de drainage externe chirurgical par rapport à la réalisation de la première endoscopie était de 4 jours pour $\mathrm{P} 2$ et 9 jours pour P1.

Nous n'avons pas eu recours à la nutrition parentérale lors de la période P2, alors que $14.7 \%$ des patients ont bénéficié de ce support nutritionnel durant la période P1. Le nombre moyen d'endoscopies avec mise en place de PDQC était de 1.3 pour P2 et de 1.9 pour P1 ( $\mathrm{p}=0.01$ ). Le taux de migration symptomatique ayant nécessité une nouvelle pose de PDQC était de $6.2 \%$ pour $\mathrm{P} 2$ et de $14.7 \%$ pour $\mathrm{P} 1$ ( $\mathrm{p}=\mathrm{NS})$.

La durée médiane d'hospitalisation globale était de 16 jours pour P2 (IQR 11.75-26) et de 30 jours pour P1 (IQR 16.25-45.75) ( $\mathrm{p}=0.0002$ ). Le taux et la durée médiane d'hospitalisation en réanimation étaient respectivement, pour $\mathrm{P} 2$ et $\mathrm{P} 1$, de $20.8 \%$ pour $\mathrm{P} 2$ et 11.5 jours (IQR 4.519.5 ) et de $52.9 \%$ et 16 jours (IQR 7.25-21.75) ( $\mathrm{p}=0.04$ et $\mathrm{p}=0.005)$, (Figure 6).

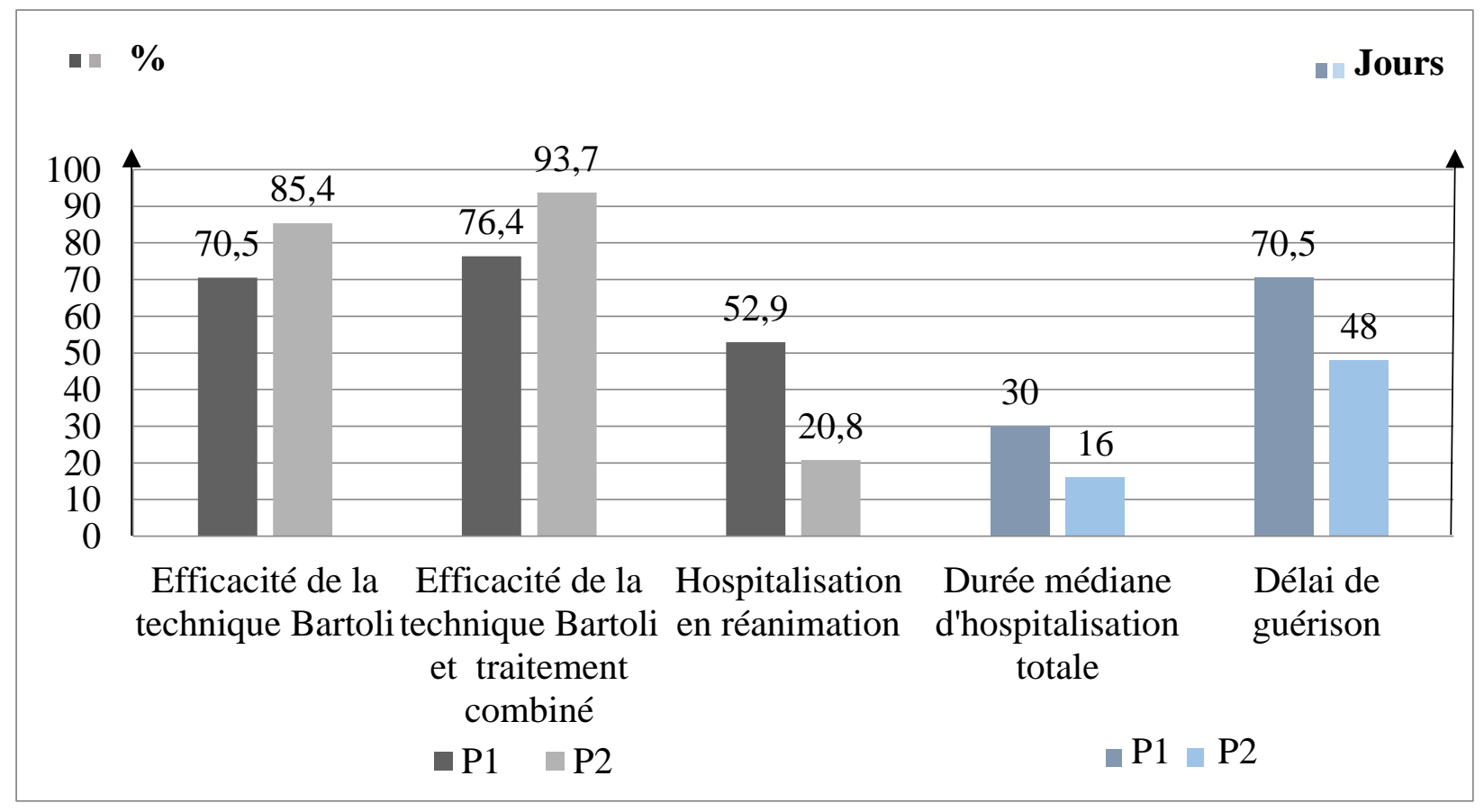

Figure 6 : Evaluation comparative de la technique Bartoli pour les périodes P1 et P2. 


\begin{tabular}{|c|c|c|c|c|}
\hline & $\begin{array}{c}\text { PT } \\
\mathrm{n}=82\end{array}$ & $\begin{array}{c}\mathbf{P 1} \\
\mathbf{n}=34\end{array}$ & $\begin{array}{c}\mathrm{P2} \\
\mathrm{n}=48\end{array}$ & $\mathbf{p}$ \\
\hline Efficacité de la technique Bartoli (n, \%) & $64(79.2)$ & $23(70.2)$ & $41(85.4)$ & 0.02 \\
\hline $\begin{array}{l}\text { Efficacité du traitement combiné + technique Bartoli } \\
(\mathrm{n}, \%)\end{array}$ & $71(86.6)$ & $25(76.5)$ & $45(93.7)$ & 0.02 \\
\hline Efficacité tout traitements endoscopiques (n, \%) & $80(97.6)$ & $33(97.1)$ & $47(97.9)$ & NS \\
\hline Succès technique (n, \%) & $81(98.8)$ & $\begin{array}{c}34 \\
(100)\end{array}$ & $47(97.9)$ & NS \\
\hline Délai de guérison (jours, IQR) & $\begin{array}{l}58(46- \\
76)\end{array}$ & $\begin{array}{l}70.5(57.5- \\
85)\end{array}$ & $\begin{array}{l}48(42.25- \\
69)\end{array}$ & 0.0017 \\
\hline Drainage endoscopique premier (n, \%) & $30(36.6)$ & $10(29.4)$ & $20(41.7)$ & NS \\
\hline Drainage radiologique premier $(\mathrm{n}, \%)$ & $\begin{array}{c}10 \\
(12.2)\end{array}$ & $\begin{array}{c}6 \\
(17.7)\end{array}$ & $\begin{array}{c}4 \\
(8.3)\end{array}$ & NS \\
\hline Durée du drainage externe radiologique (jours, IQR) & $\begin{array}{l}7(3.5- \\
9.75)\end{array}$ & $7(5.5-8.5)$ & $\begin{array}{l}6.5(2.5- \\
11.75)\end{array}$ & NS \\
\hline Drainage chirurgical premier $(\mathrm{n}, \%)$ & $41(51.2)$ & $18(52.9)$ & $\begin{array}{c}24 \\
(50)\end{array}$ & NS \\
\hline Durée du drainage externe chirurgical (jours, IQR) & $4(3-11)$ & $9(4-15)$ & $4(3-6.75)$ & 0.0009 \\
\hline Reprise post endoscopie (n, \%) & $9(11)$ & $5(14.7)$ & $3(8.3)$ & NS \\
\hline $\begin{array}{l}\text { Nombre moyen d'endoscopie avec pose de PDQC } \\
\text { (extrêmes) }\end{array}$ & $1.6(1-7)$ & $1.9(1-7)$ & $1.3(1-4)$ & 0.01 \\
\hline Nombre médian d'endoscopie thérapeutique (IQR) & $2(2-3.75)$ & $2(2-4.75)$ & $1(2-3)$ & 0.0004 \\
\hline Tolérance (n, \%) & $80(97.6)$ & $\begin{array}{l}33 \\
(97)\end{array}$ & $47(97.9)$ & NS \\
\hline Migration (n, \%) & $16(19.5)$ & $\begin{array}{c}8 \\
(23.5)\end{array}$ & $\begin{array}{c}8 \\
(16.6)\end{array}$ & NS \\
\hline Migration symptomatique (n, \%) & $\begin{array}{c}8 \\
(9.7)\end{array}$ & $\begin{array}{c}5 \\
(14.7)\end{array}$ & $\begin{array}{c}3 \\
(6.2)\end{array}$ & NS \\
\hline Perforation $(\mathrm{n}, \%)$ & $3(3.6)$ & $1(2.9)$ & $2(4.2)$ & NS \\
\hline Hémorragie (n, \%) & 0 & 0 & 0 & NS \\
\hline Décès $(\mathrm{n}, \%)$ & 0 & 0 & 0 & NS \\
\hline $\begin{array}{l}\text { Support nutritionnel entérale (SNJ+ jéjunostomie) (n, } \\
\%)\end{array}$ & $72(87.8)$ & $29(85.3)$ & $43(89.6)$ & NS \\
\hline Support nutritionnel parentéral (n, \%) & $5(6.1)$ & $5(14.7)$ & 0 & 0.01 \\
\hline Absence de support nutritionnel (n, \%) & $7(8.5)$ & $2(5.9)$ & $5(10.4)$ & NS \\
\hline Nombre médian d'hospitalisation (IQR) & $2(2-3)$ & $2(2-3)$ & $2(2-3)$ & NS \\
\hline Durée médiane d'hospitalisation totale (jours, IQR) & $\begin{array}{l}18.5(13- \\
31)\end{array}$ & $\begin{array}{l}30(16.25- \\
\quad 45.75\end{array}$ & $\begin{array}{l}16(11.75- \\
26)\end{array}$ & 0.0002 \\
\hline Hospitalisation en réanimation (n, \%) & $28(34.1)$ & $18(52.9)$ & $10(20.8)$ & 0.04 \\
\hline $\begin{array}{l}\text { Durée médiane d'hospitalisation en réanimation } \\
\text { (jours, IQR) }\end{array}$ & $\begin{array}{l}15(5.5- \\
21)\end{array}$ & $\begin{array}{c}16(7.25- \\
21.75)\end{array}$ & $\begin{array}{c}11.5 \\
(4.5-19.5)\end{array}$ & 0.005 \\
\hline $\begin{array}{l}\text { Chirurgie radicale par anastomose gastro-jéjunale au } \\
\text { contact de la FG (n, \%) }\end{array}$ & $2(2.4)$ & $1(2.9)$ & $1(2.1)$ & NS \\
\hline
\end{tabular}

Tableau 3: Résultats pour la période PT, P1 et P2 et comparaison des périodes P1 et P2

PT PDQC : prothèses double queue de cochon, $F G$ : fistule gastrique 


\subsection{Analyse univariée}

Après analyse univariée, il n'a pas été retrouvé de facteurs indépendants influençant le succès et l'échec de la technique Bartoli (Tableau 4).

\begin{tabular}{|l|c|c|c|}
\hline Variables & Odds ratio & $\begin{array}{c}\text { Intervalle de } \\
\text { confiance à 95\% }\end{array}$ & p value \\
\hline Age & 1.09 & $0.94-1.12$ & 0.29 \\
\hline Sexe & 1.45 & $0.55-5.08$ & 0.31 \\
\hline IMC & 0.96 & $0.81-1.08$ & 0.27 \\
\hline HTA & 1.98 & $0.14-14.9$ & 0.54 \\
\hline Diabète & 0.65 & $0.09-2.76$ & 0.25 \\
\hline Dyslipidémie & 1.9 & $0.22-11.97$ & 0.66 \\
\hline SAOS & 1.34 & $0.32-5.69$ & 0.69 \\
\hline $\begin{array}{l}\text { Syndrome } \\
\text { métabolique }\end{array}$ & 0.34 & $0.13-1.32$ & 0.35 \\
\hline ATCD chirurgical & 0.76 & $0.47-9.91$ & 0.71 \\
\hline $\begin{array}{l}\text { Délai diagnostic de la } \\
\text { fistule }\end{array}$ & 1.21 & $0.65-2.38$ & 0.24 \\
\hline Taille de la fistule & 0.61 & $0.09-6.1$ & 0.72 \\
\hline Leucocytes & 1 & $1-1$ & 0.69 \\
\hline CRP & 1 & $0.98-1.02$ & 0.99 \\
\hline Albumine & 1.37 & $0.92-1.98$ & 0.27 \\
\hline Support nutritionnel & 1.09 & $0.10-7.52$ & 0.93 \\
\hline Nombre de PDQC & 1.03 & $0.50-6.01$ & 0.78 \\
\hline Migration des PDQC & 2.11 & $0.09-9.28$ & 0.7 \\
\hline Sténose & 1.92 & $0.25-15.87$ & 0.51 \\
\hline
\end{tabular}

Tableau 4 : Analyse univariée

IMC : indice de masse corporelle, HTA : hypertension artérielle, SAOS : syndrome d'apnée obstructive du sommeil, ATCD : antécédents, CRP : C reactive protein, $P D Q C$ : prothèses double queue de cochon. 


\section{DISCUSSION}

La FG post SG est la complication la plus redoutée du fait de sa morbidité. Elle est aussi responsable de la majorité des décès liés à la SG. Actuellement, aucun consensus n'existe pour la gestion des FG post SG. Les experts s'accordent qu'en cas de de sepsis non contrôlé, une reprise chirurgicale ou un drainage radiologique est nécessaire (77). Le traitement endoscopique associé à un support nutritionnel, un traitement par IPP et une antibiothérapie adaptée est ensuite initié de manière complémentaire au drainage externe ou en première intention en cas d'absence de sepsis et en cas de collection bien individualisée.

Les SEMS ont été largement utilisés avec des résultats variables, un mauvais profil de tolérance nécessitant souvent leur remplacement voire leur ablation augmentant ainsi le nombre d'endoscopies sous anesthésie générale, un taux de migration important et des complications hémorragiques potentiellement fatales (66). Les PDQC permettent le drainage interne des FG post SG, dont le primum movens est leur rôle dans le drainage des pseudokystes pancréatiques (75). L'hypothèse physiopathologique repose sur une cicatrisation dirigée de l'extérieur vers l'intérieur de par les différences de pression entre la cavité gastrique, la cavité péritonéale et l'air. Les PDQC maintiennent ouvert le trajet de la FG entre la lumière de l'estomac et l'espace infecté, permettent un nettoyage/drainage de la collection et aboutissent à une pseudo-cavité indemne d'infection. Notre étude renforce l'idée que l'utilisation des PDQC offre un avantage par rapport à l'utilisation des SEMS par la simplicité du geste endoscopique, le profil de tolérance, un taux de migration et de complication moindre et une efficacité sur la cicatrisation moins aléatoire.

Le drainage par PDQC a été décrit pour la première fois dans les FG post SG par Pequignot et al. (78), et rapportait une technique sûre, efficace et une durée d'hospitalisation réduite. Donatelli et al et Bouchard et al. ont rapporté respectivement un succès technique de $78.2 \%$ et $78.8 \%$ lors de la réalisation d'un drainage interne de fistules digestives supérieures par l'utilisation de PDQC (79, 80). Néanmoins, l'utilisation des PDQC a été évaluée rétrospectivement sur de faibles effectifs (respectivement 64 et 33 patients) avec des caractéristiques hétérogènes concernant le site de la fistule (proximal ou distal), le type de chirurgie initiale (SG ou by pass), le nombre et la taille des PDQC, la prise en charge des sténoses associées et les modalités de gestion péri-endoscopique. L'efficacité de la technique par pose de PDQC a été évaluée chez des patients ayant déjà reçu un traitement endoscopique dans 57.6\% des cas dans l'étude de Bouchard et al. Dans l'étude de Donatelli et al, 3 patients 
ont été exclus de l'analyse finale (deux décès et un échec technique) source de biais et d'une méthodologie contestable. De plus, le critère de jugement principal pour cette dernière étude n'était pas clairement défini, tout comme l'efficacité du traitement endoscopique qui reposait sur un critère uniquement radiologique. Enfin, des données importantes n'étaient pas mentionnées comme le nombre et la durée totale d'hospitalisation, le nombre d'endoscopies, la durée du drainage externe, ainsi que le recours à une unité de soins intensifs, qui ont un impact sur la qualité de la prise en charge globale du patient.

A notre connaissance, notre cohorte est la plus importante, la plus complète décrivant l'utilisation de PDQC dans le traitement des FG post SG, et ayant sélectionné la population sur des critères d'inclusion stricts. Notre cohorte comportait uniquement des patients ayant une FG proximale post SG et ayant bénéficié d'un traitement premier par pose de PDQC. L'harmonisation de notre population, l'utilisation d'un critère de jugement principal unique et non équivoque, une définition composite simple de l'efficacité basée sur la reprise de l'alimentation par voie orale en l'absence de signe de sepsis, de modules de drainage interne ou externe et de collection radiologique, et une description claire de la technique endoscopique utilisée, nous a permis une analyse plus pertinente de l'efficacité de la technique Bartoli et de renforcer la méthodologie, malgré les biais liés au caractère rétrospectif de l'analyse.

Nous rapportons un taux d'efficacité sur la cicatrisation de $79.2 \%$ sur 82 patients et de $85.4 \%$ après avoir standardisé les modalités de la procédure lors de la période P2. L'utilisation associée d'une technique dérivée, combinant des PDQC posées au travers d'un SEMS, permettait d'aboutir à un taux d'efficacité de $86.5 \%$ pour la période PT et de $93.7 \%$ pour la période $\mathrm{P} 2$. Ce traitement combiné consistait en la mise en place d'une prothèse métallique couverte œsophagienne pour couvrir la fistule notamment lorsque celle-ci était large et de traiter une sténose ou un twist le cas échéant, associée à la mise en place de 2 PDQC au travers du SEMS afin de drainer la fistule, comme cela a été décrit par Rebibo et al (81).

Aucune PDQC n'a été retirée pour mauvaise tolérance. Seuls deux patients $(2.4 \%)$ se sont plaints de douleur et de nausées en post endoscopie et ont rapidement été soulagés par un traitement symptomatique. Le taux de migration digestive était de $19.5 \%(n=16)$, mais seuls 9.7\% $(\mathrm{n}=8)$ des patients ont bénéficié d'une nouvelle endoscopie pour mise en place à nouveau de PDQC devant le caractère symptomatique de la migration. Deux cas de migration intra splénique se compliquant d'abcès ont été rapportés $(82,83)$. Dans notre expérience, nous 
n'avons déploré aucune migration intra péritonéale de PDQC, cette complication étant probablement exceptionnelle, facilitée par une grande taille de la fistule autorisant la migration intra péritonéale de la boucle gastrique de la PDQC.

Contrairement à la prise en charge par SEMS qui nécessite une gestion hospitalière du drainage externe de l'abcès au contact de la fistule pendant la durée du sepsis; la prise en charge par PDQC autorise des durées courtes de drainage externe entre quatre et sept jours selon l'abord, permet des durées d'hospitalisation raccourcies et un meilleur confort pour le patient, suggérant ainsi un bénéfice sur la qualité de vie. De plus, la réalisation d'un drainage interne premier a permis de surseoir à un drainage externe pour $36.6 \%$ des patients lors de la période PT et $41.7 \%$ des patients lors de la période P2. Malgré l'absence de validation, une évaluation médico-économique bénéfique est prévisible.

L'expérience du CHU d'Amiens concernant l'utilisation des PDQC dans les FG post SG remonte à 2005, initialement en seconde intention après de multiples échecs de la pose de SEMS pour couvrir la FG. L'amélioration progressive de la gestion médicale, endoscopique et chirurgicale des PDQC lors de la période P1 a permis une harmonisation de la technique Bartoli dès novembre 2013. Les résultats de la période P2, portant sur 48 patients, sont un meilleur reflet de notre prise en charge actuelle des FG post SG avec des résultats significativement meilleurs en matière d'efficacité, de gestion péri-endoscopique et de modalités d'hospitalisation. Ces résultats témoignent de la simplicité et de l'efficacité de la technique Bartoli sur la prise en charge des FG liées aux SG, lorsque celle-ci est standardisée.

Aucun facteur de risque d'échec n'a été identifié, probablement du fait du caractère rétrospectif de notre étude, d'un manque de puissance et de l'évolution de la prise en charge de la procédure entre les périodes P1 et P2. Néanmoins, dans notre expérience, les principaux facteurs d'échec étaient la présence d'une large fistule et la présence d'une sténose ou d'un «twist gastrique ». Ceux-ci peuvent être responsables d'une migration rapide des PDQC ou entretenir le mécanisme pathogénique de la fistule en l'absence de traitement étiologique. Ces patients n'ont majoritairement pas été inclus lors de la période P2, car ils ont bénéficié en première intention du traitement combiné, ce qui explique le faible taux de sténose. L'évaluation de la taille des FG n'est pas un élément fiable dans notre étude, car soumise à la subjectivité des différents endoscopistes. Malgré la pratique fréquente de la technique combinée, nous avons décidé de ne pas inclure ces patients ou de les considérer en échec de la 
technique Bartoli pour homogénéiser la procédure et rendre plus pertinente l'évaluation de la procédure utilisant des PDQC.

La nouvelle approche proposée par la technique Bartoli n'est pas dépendante du caractère précoce ou tardif de l'apparition de la FG, contrairement aux prises en charge actuellement préconisées, reposant sur des délais de survenue variables et sur des avis d'expert. Notre approche plus pragmatique est basée sur la tolérance clinique du sepsis, la présence d'une collection bien délimitée à la tomodensitométrie et l'évaluation endoscopique de la taille de la fistule et de la présence d'une sténose gastrique ou non. La technique Bartoli permet de simplifier la prise en charge «dans la vraie vie » des FG post SG et nous permet de proposer un algorithme de prise en charge (Figure 7).

Notre étude comporte certaines limites. Malgré un recueil prospectif de nombreuses données, notre analyse est rétrospective avec une prise en charge mono centrique des FG. De plus, il s'agit d'une étude de cohorte ne comparant pas la technique Bartoli à une prise en charge par SEMS pour couvrir la fistule. Néanmoins, à notre connaissance, il n'existe aucune évaluation prospective comparative de la prise en charge des FG post SG. Une évaluation prospective multi centrique est nécessaire pour confirmer ces résultats et une évaluation est en cours d'élaboration au CHU d'Amiens sous l'égide du GRAPHE.

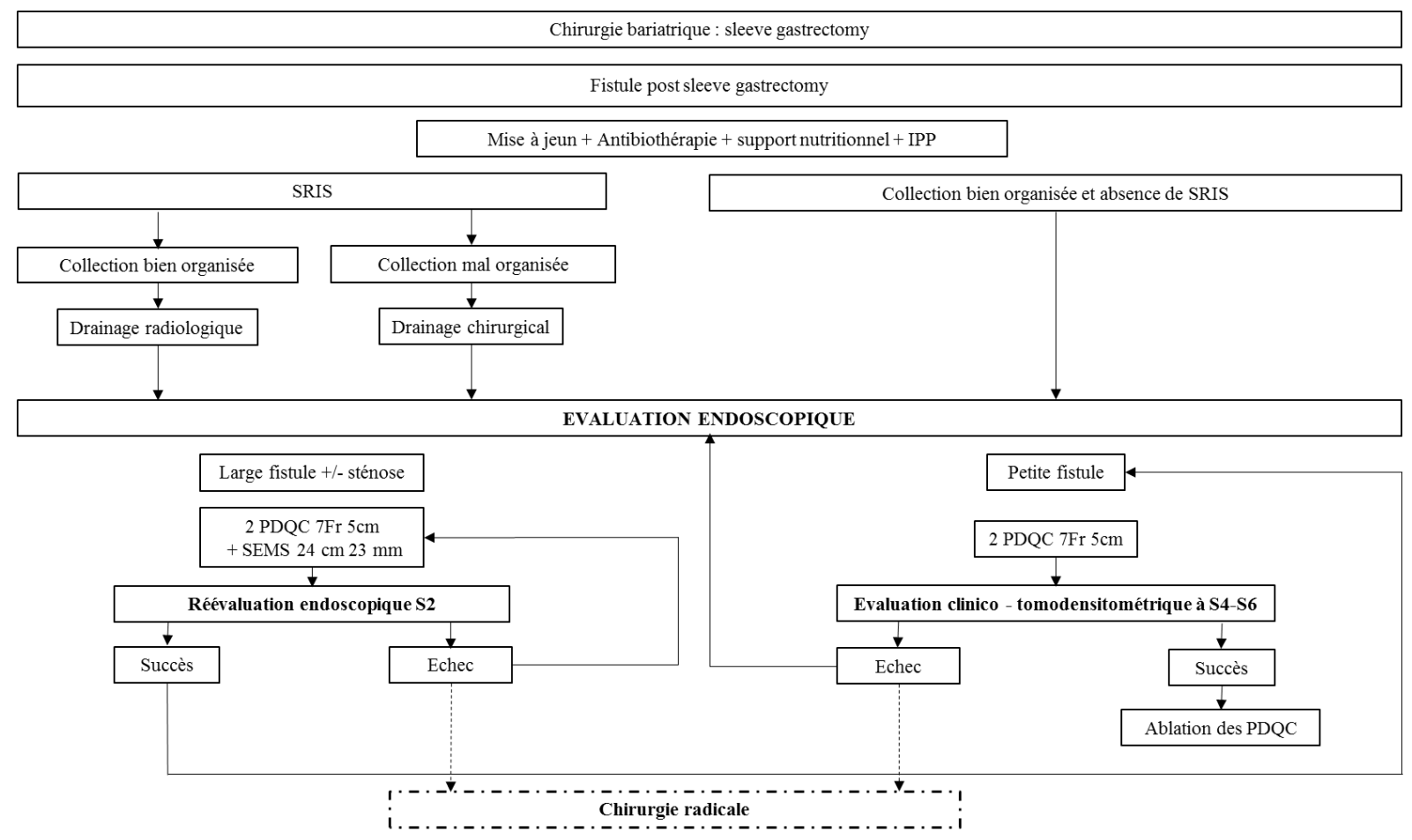

Figure 7 : Prise en charge de la FG post SG 


\section{CONCLUSION}

La prise en charge d'une FG post SG par drainage interne de type PDQC est efficace, cliniquement bien tolérée, avec une faible morbidité. Cette technique permet une gestion des modules de drainage externe simple, un délai de guérison et une durée d'hospitalisation courts, et un faible taux de chirurgie radicale. Cependant cette technique nécessite d'être évaluée par une étude prospective multicentrique. 


\section{ANNEXES}

\section{Annexe 1 : ballon intra gastrique}

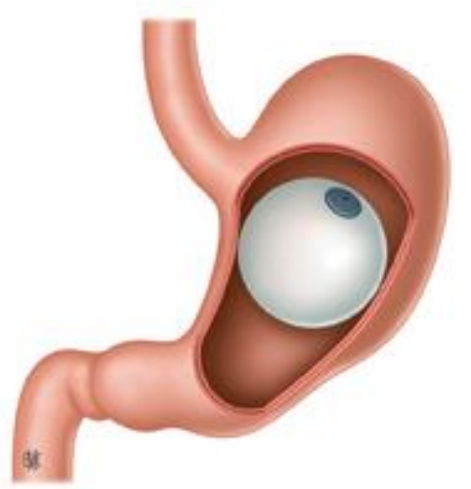

Annexe 2 : Dispositifs d'agrafage transoral TOGA ${ }^{\circledR}$ : dispositif de gastroplastie par voie transorale

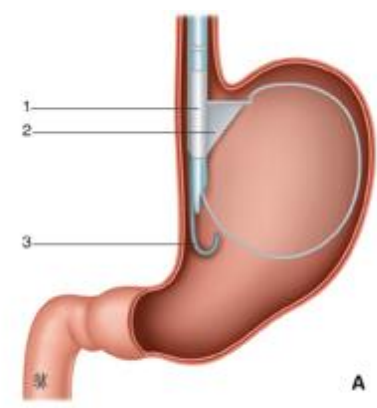

A : Un fil guide en forme de voile est déployé durant la procédure afin de maintenir l'estomac dans la bonne position.

1. Agrafeuse endoscopique. 2. Endoscope. 3. Zone triangulaire de muqueuse aspirée et agrafée délimitant le compartiment gastrique.

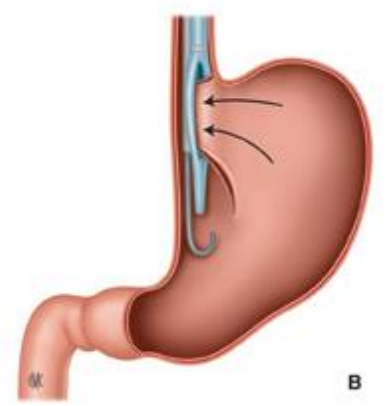

B : Aspiration et agrafages successifs de la muqueuse gastrique péri cardiale dans le canal opérateur réalisant ainsi une poche de restriction. 


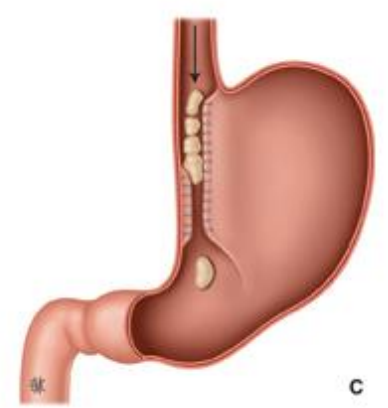

$\mathrm{C}:$ Aspect en fin de procédure.

Annexe 3 : Dispositifs de sutures gastriques transorales : EndoCinch ${ }^{\mathrm{TM}}$ et RESTORe ${ }^{\mathrm{TM}}$.
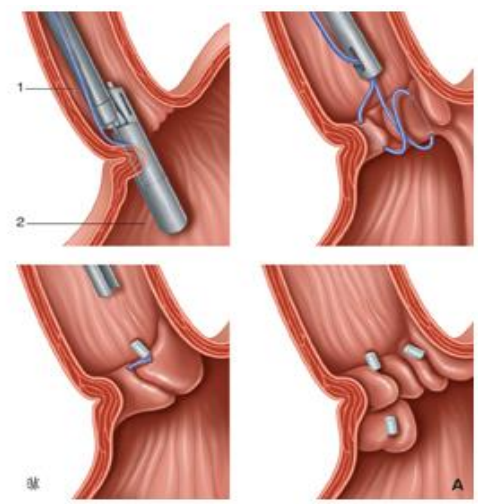

A : EndoCinch ${ }^{\mathrm{TM}}$. Aspiration de la muqueuse gastrique dans le canal opérateur de l'endoscope et suture transfixiante de la muqueuse aspirée; les segments muqueux suturés sont ensuite réunis délimitant un compartiment gastrique. 1. Esophage ; 2. Estomac.
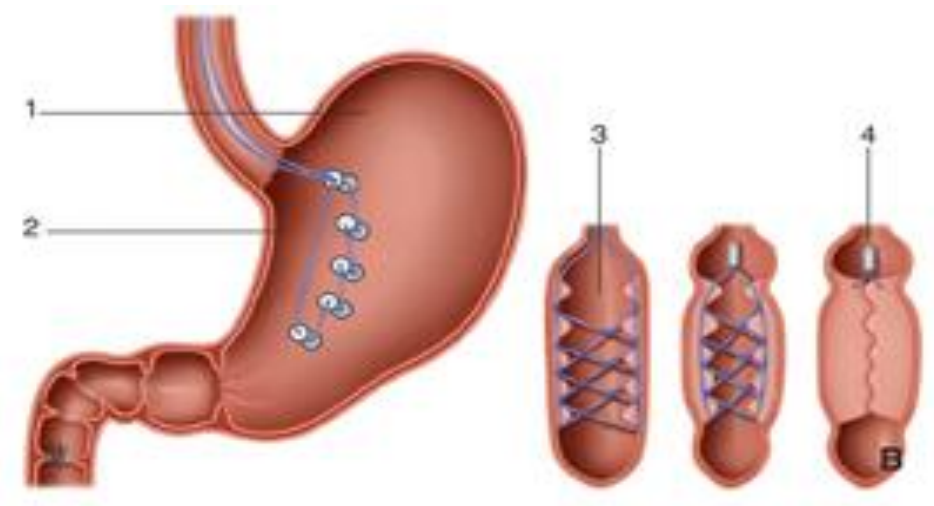

B : RESTORe ${ }^{\mathrm{TM}}$. Localisation des différentes zones de plicature : depuis le fundus (1) puis sur les plans antérieur et postérieur le long de la petite courbure gastrique (2) : les fils de la plicature sont ensuite serrés délimitant ainsi un compartiment gastrique (3) : aspect en fin de procédure (4). 
Annexe 4 : Implants malabsorptifs : ValenTx et EndoBarrier

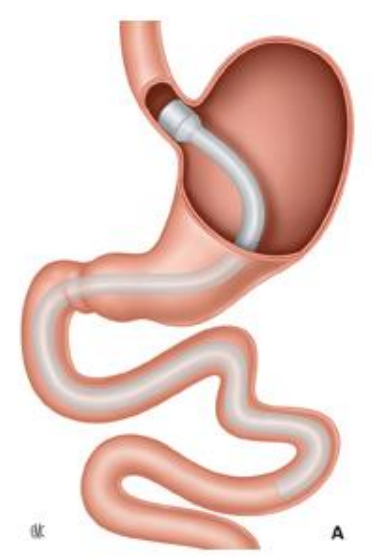

A : valenTx.

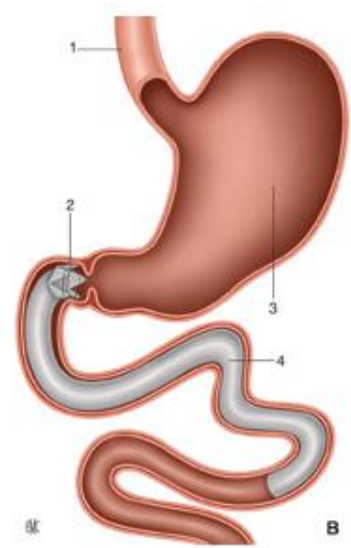

B : Endobarrier ; 1. Esophage ; 2. duodénum ; 3. estomac ; 4. gaine de l'EndoBarrier ${ }^{\circledR}$.

\section{Annexe 5 : Sleeve gastrectomy}

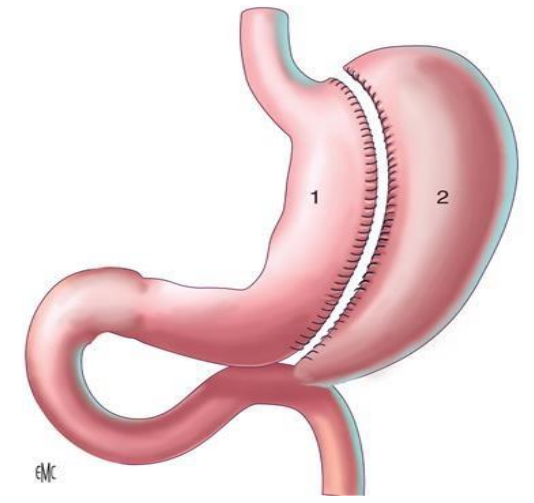

1. Manchon gastrique 2. Estomac réséqué. 


\section{Annexe 6 :}

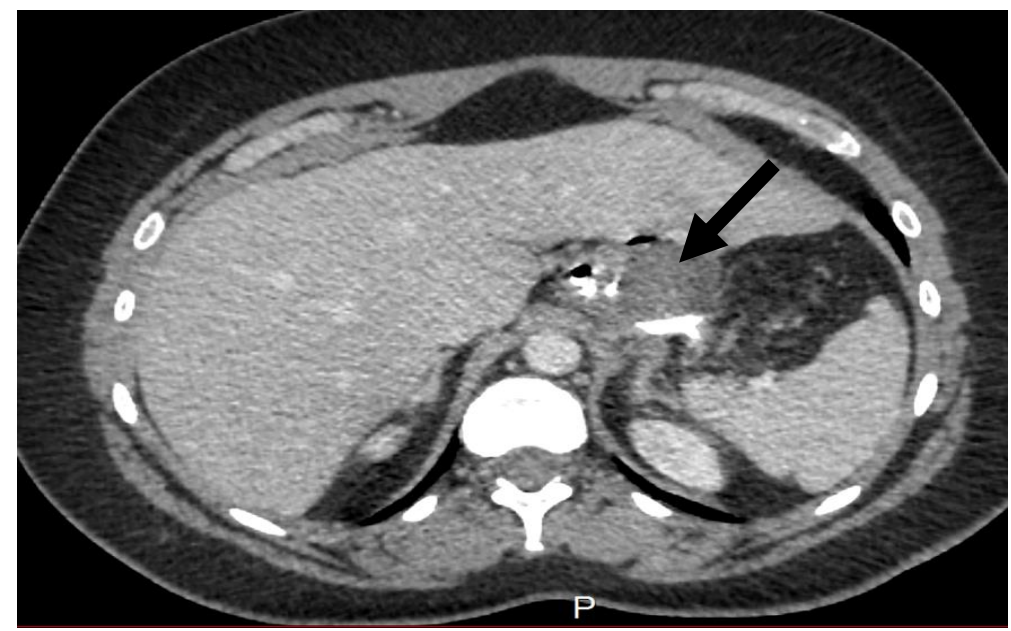

Coupe scannographique transversale d'une tomodensitométrie abdominale avec injection de produit de contraste et opacification haute. Opacification de la collection péri gastrique témoignant d'une fistule gastrique.

\section{Annexe 7 :}

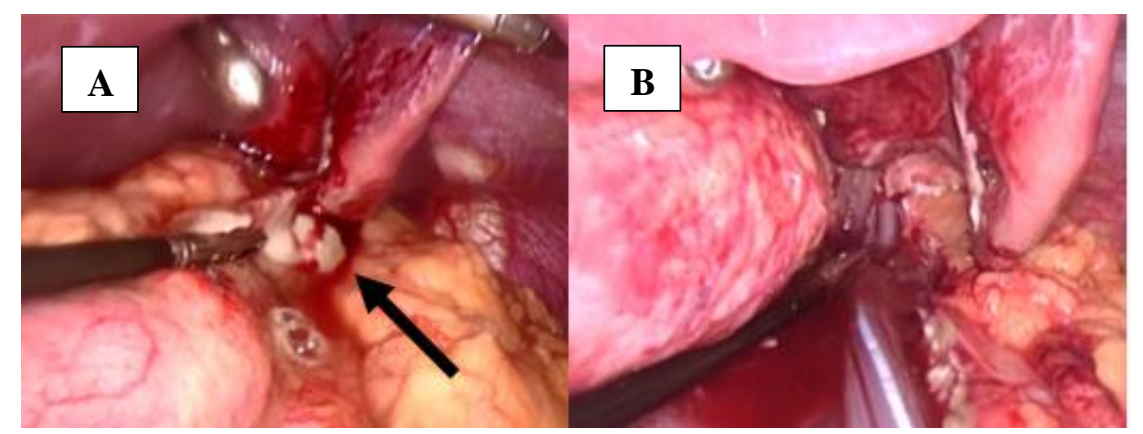

Photographie per opératoire sous cœlioscopie, visualisant l'abcès au contact de la partie proximale de l'estomac (A), et mise en place de module de drainage externe (B).

\section{Annexe 8 :}

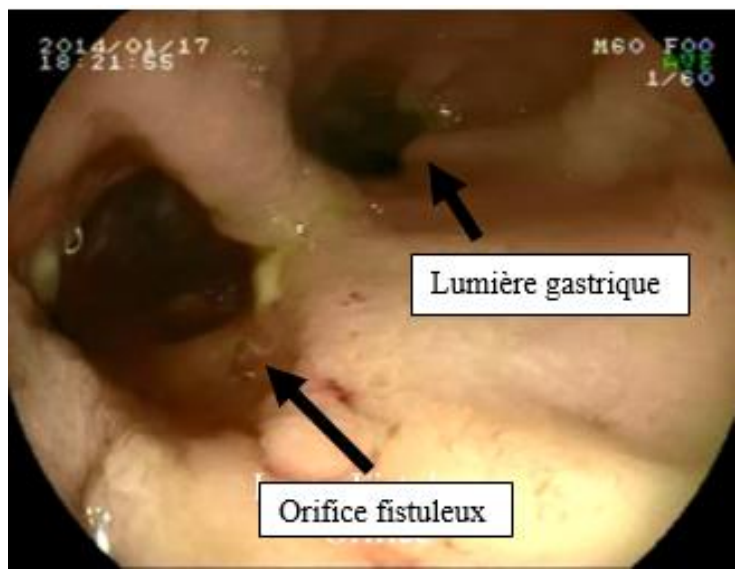

Photographie endoscopique visualisant une large fistule post SG. 


\section{Annexe 9 :}

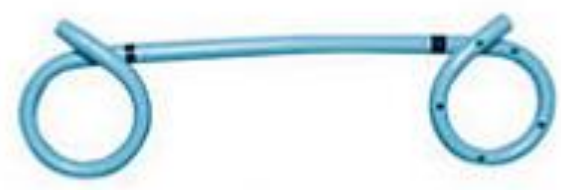

Prothèse double queue de cochon $7 F r 5 \mathrm{~cm}$.

Annexe 10 :

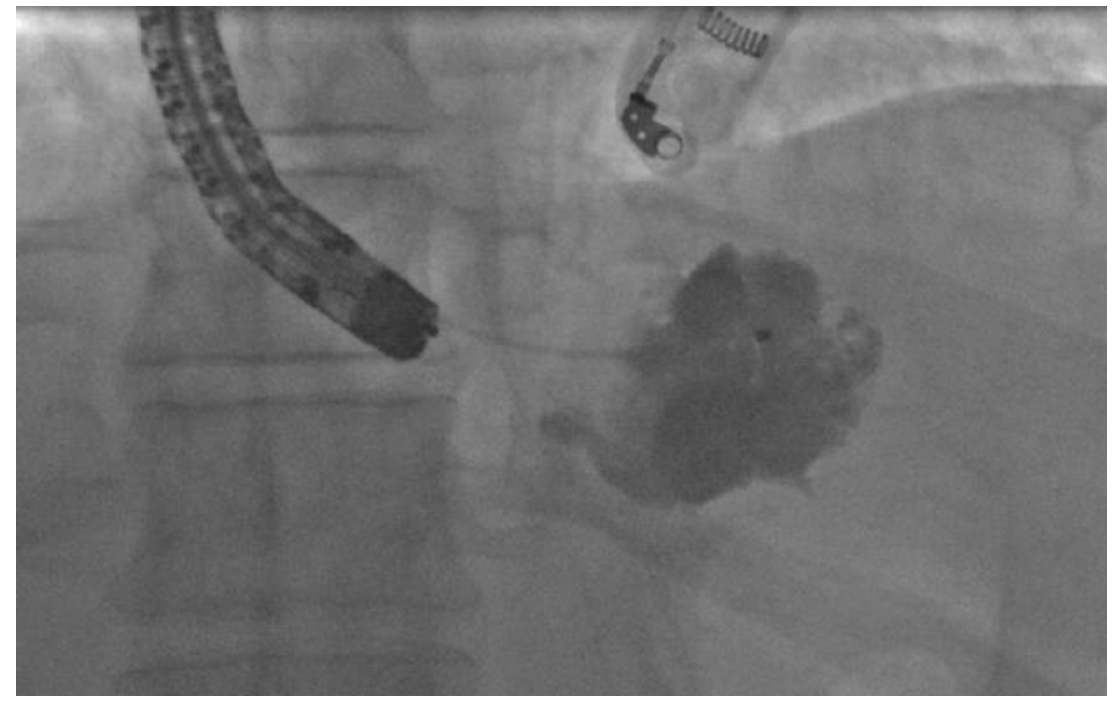

Image fluoroscopique d'une opacification de la cavité abcédée après cathétérisme de la FG.

Annexe 11 :

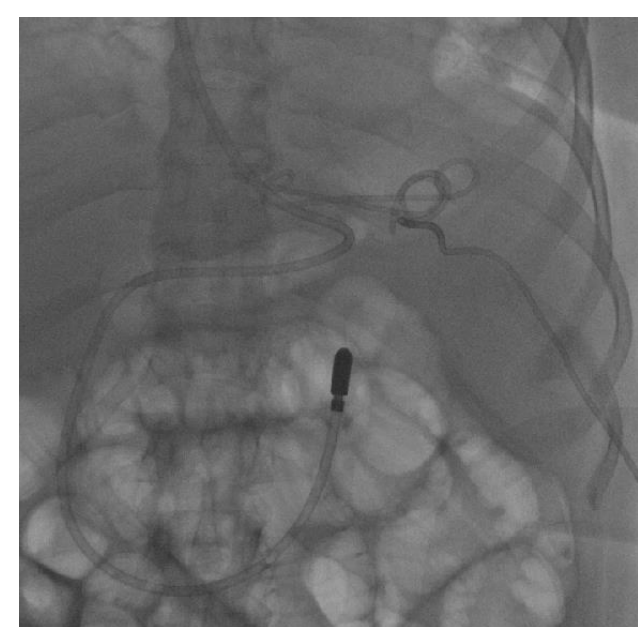

Image fluoroscopique en fin de procédure : 2PDQC, le drainage externe au contact et une SNJ. 


\section{Annexe 12 :}

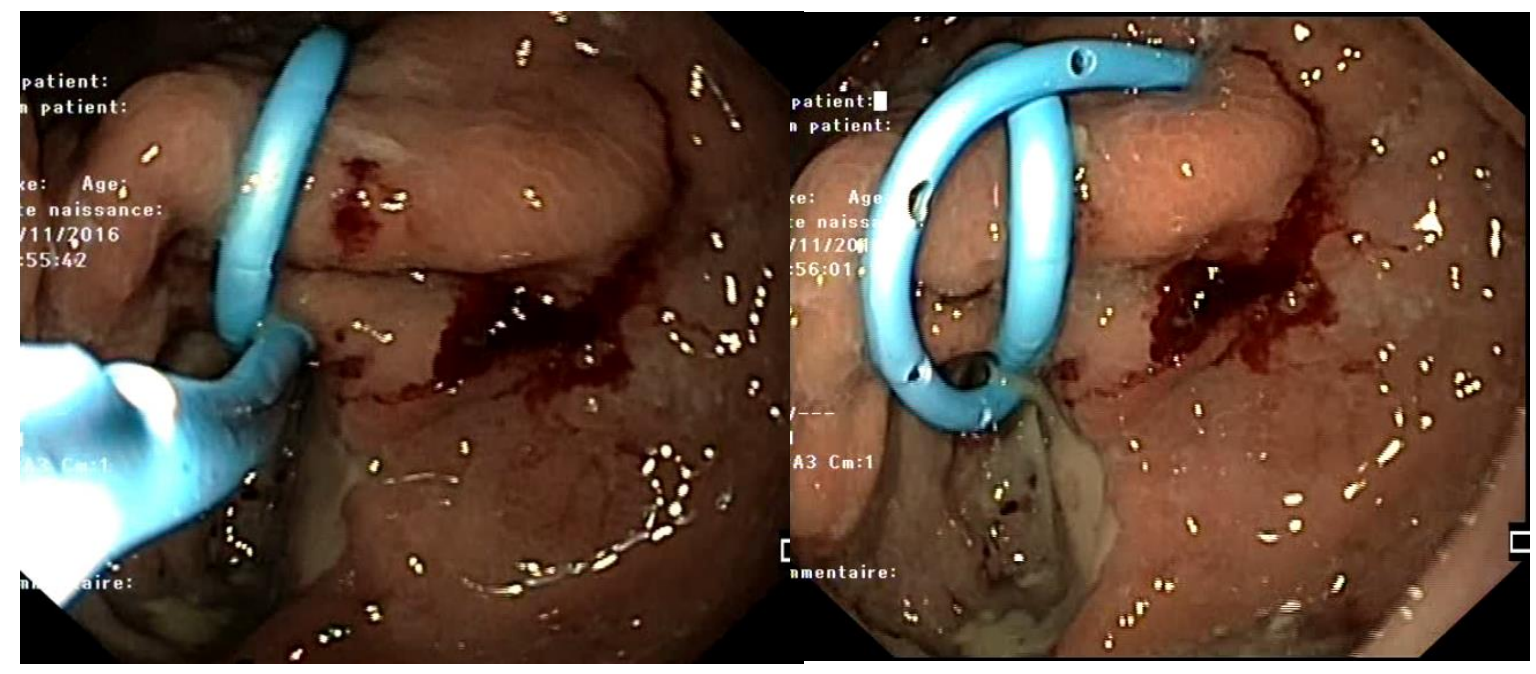

Photographie per endoscopique de 2 PDQC dans la FG post SG.

\section{Annexe 13 :}

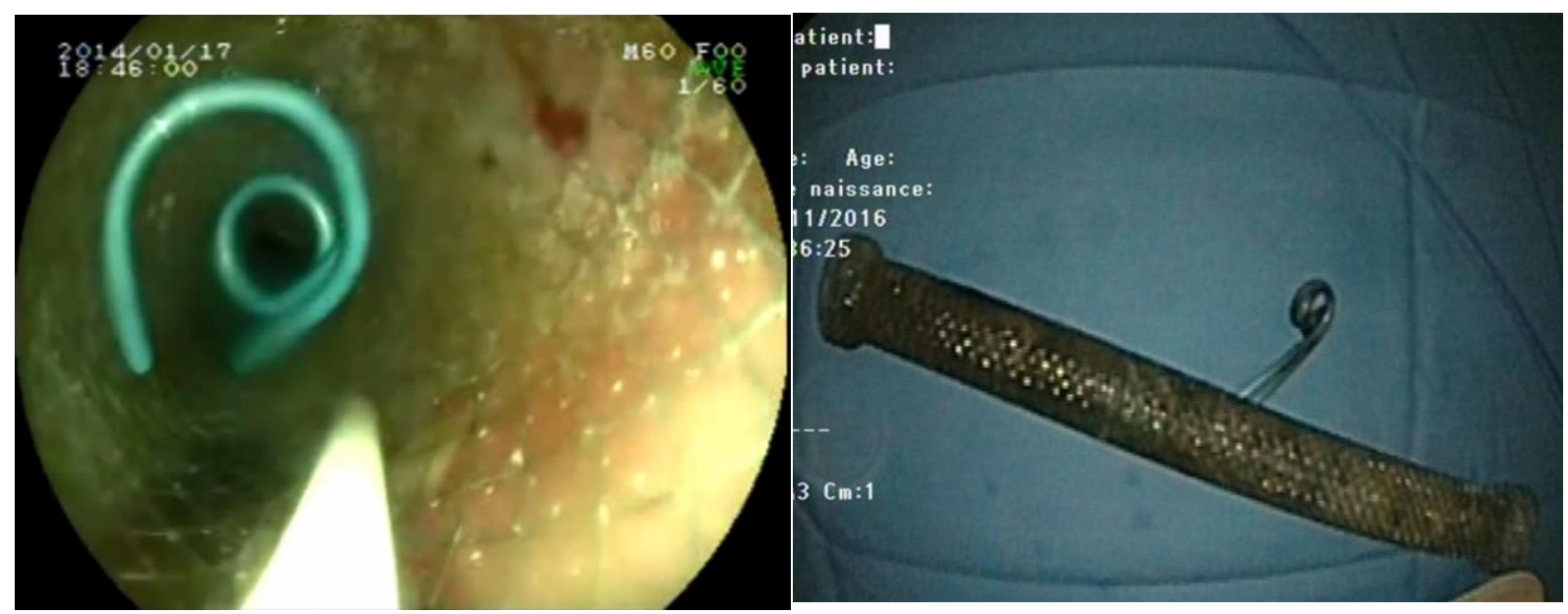

Photographie endoscopique du traitement combiné : SEMS + 2 PDQC. 


\section{REFERENCES BIBLIOGRAPHIQUES}

1. Enquête oBépi 2012. [Internet] Enquête épidémiologique nationale sur le surpoids et l'obésité [cité 27 févr 2017]. Disponible sur:

http://www.roche.fr/content/dam/roche_france/fr_FR/doc/obepi_2012.pdf

2. Bray GA. Health hazards of obesity. Endocrinol Metab Clin North Am.1996; 25:907-19.

3. Oppert JM, Charles MA, Thibult N, Guy-Grand B, Eschwège E, Ducimetière P.Anthropometric estimates of muscle and fat mass in relation to cardiac and cancer mortality in men: the Paris Prospective Study. Am J Clin Nutr. 2002 ; 75:1107-13.

4. Charles M-A. Épidémiologie des obésités de l'adulte. EMC

5. Romon I, Rey G, Mandereau-Bruno L, Weill A, Jougla E, Eschwège E, et al. The excess mortality related to cardiovascular diseases and cancer among adults pharmacologically treated for diabetes-the 2001-2006 ENTRED cohort. Diabet Med. 2014; 31:946- 53.

6. Denke MA,Sempos CT, Grundy SM. Excess body weight. An underrecognized contributor to high blood cholesterol levels in white American men. Arch Intern Med. 1993 ; 153:1093-103.

7. Jenny-Avital ER. Obesity and the risk of heart failure. N Engl J Med. 2002; 347:1887-9; author reply 1887-9.

8. Guh DP, Zhang W, Bansback N, Amarsi Z, Birmingham CL, Anis AH. The incidence of comorbidities related to obesity and overweight: A systematic review and meta-analysis. BMC Public Health. 2009; 9:88

9. Lakka HM, Laaksonen DE, Lakka TA, Niskanen LK, Kumpusalo E, Tuomilehto J, Salonen JT. The metabolic syndrome and total and cardiovascular disease mortality in middle-aged men. JAMA. $2002 ; 288: 2709-16$.

10. Prospective Studies Collaboration. Body-mass index and cause-specific mortality in 900000 adults: collaborative analyses of 57 prospective studies. The Lancet. 2009; 373:1083-96.

11. Renehan AG, Tyson M, Egger M, Heller RF, Zwahlen M. Body-mass index and incidence of cancer: a systematic review and meta-analysis of prospective observational studies. The Lancet. 2008; 371:569- 78.

12. Obesity: preventing and managing the global epidemic. Report of a WHO consultation. World Health Organ Tech Rep Ser. 2000; 894:i-xii, 1-253.

13. Felson DT, Anderson JJ, Naimark A, Walker AM, Meenan RF. Obesity and knee osteoarthritis. The Framingham Study. Ann Intern Med. 1988; 109:18-24.

14. Cooper C, Inskip H, Croft P, Campbell L, Smith G, McLaren M, Coggon D. Individual risk factors for hip osteoarthritis: obesity, hip injury, and physical activity. Am J Epidemiol. 1998; $147: 516-22$.

15. A. Anandacoomarasam, ${ }^{\mathrm{I}}$ Caterson, $\mathrm{P}$ Sambrook, M Fransen and L March. The impact of obesity on the musculoskeletal system International Journal of Obesity (2008) 32, 211-222 
16. Marchesini G, Moscatiello S, Di Domizio S, Forlani G. Obesity-Associated Liver Disease. J Clin Endocrinol Metab. 2008; 93:s74- 80.

17. Stampfer MJ, Maclure KM, Colditz GA, Manson JE, Willett WC. Risk of symptomatic gallstones in women with severe obesity. Am J Clin Nutr. 1992; 55:652-8.

18. Bart S, Ciangura C, Thibault F, Cardot V, Richard F, Basdevant A, Chartier-Kastler E. Stress urinary incontinence and obesity. Prog Urol. 2008; 18:493-8

19. Praga M, Morales E. Obesity, proteinuria and progression of renal failure: Curr Opin Nephrol Hypertens. 2006; 15:481 - 6.

20. Navarro-Díaz M, Serra A, López D, Granada M, Bayés B, Romero R. Obesity, inflammation, and kidney disease. Kidney Int. 2008; 74:S15- 8.

21. Serra A, Romero R, Lopez D, Navarro M, Esteve A, Perez N, et al. Renal injury in the extremely obese patients with normal renal function. Kidney Int.2008; 73:947- 55.

22. Praga M, Morales E. Weight Loss and Proteinuria. In: Wolf G, éditeur. Contributions to Nephrology 2006; 151:221-9.

23. Dixon JB, Dixon ME, O'Brien PE. Depression in Association with Severe Obesity: Changes with weight loss. Arch Intern Med. 2003; 163:2058.

24. Poghosyan T, Polliand C, Bernard K, Rizk N, Valensi P, Champault G. Comparison of quality of life in morbidly obese patients and healthy volunteers. A prospective study using the GIQLI questionnaire. J Chir (Paris). 2007; 144:129-33; discussion 134.

25. Emery C, Dinet J, Lafuma A, Sermet C, Khoshnood B, Fagnani F. Évaluation du coût associé à l'obésité en France. Presse Médicale. 2007; 36:832- 40.

26. Effertz T, Engel S, Verheyen F, Linder R. The costs and consequences of obesity in Germany: a new approach from a prevalence and life-cycle perspective. Eur $\mathrm{J}$ Health Econ.2016; 17:1141- 58 .

27. Kim DD, Basu A. Estimating the Medical Care Costs of Obesity in the United States: Systematic Review, Meta-Analysis, and Empirical Analysis. Value Health. 2016; 19:602- 13.

28. Ponce J, Quebbemann BB, Patterson EJ. Prospective, randomized, multicenter study evaluating safety and efficacy of intragastric dual-balloon in obesity. Surg Obes Relat Dis. $2013 ; 9: 290-5$.

29. Fogel R, De Fogel J, Bonilla Y, De La Fuente R. Clinical experience of transoral suturing for an endoluminal vertical gastroplasty: 1-year follow-up in 64 patients. Gastrointest Endosc. 2008;68:51- 8 .

30. Familiari P, Costamagna G, Bléro D, Le Moine O, Perri V, Boskoski I, et al. Transoral gastroplasty for morbid obesity: a multicenter trial with a 1-year outcome. Gastrointest Endosc. 2011; 74:1248- 58. 
31. Biertho L, Hould F-S, Lebel S, Biron S. Transoral endoscopic restrictive implant system: a new endoscopic technique for the treatment of obesity. Surg Obes Relat Dis.2010;6:203- 5.

32. Sandler BJ, Rumbaut R, Paul Swain C, Torres G, Morales L, Gonzales L, et al. Human experience with an endoluminal, endoscopic, gastrojejunal bypass sleeve. Surg Endosc. 2011; $25: 3028-33$.

33. Schauer PR, Kashyap SR, Wolski K, Brethauer SA, Kirwan JP, Pothier CE, et al. Bariatric Surgery versus Intensive Medical Therapy in Obese Patients with Diabetes. N Engl J Med. 2012; 366:1567- 76 .

34. Sjöström L, Lindroos AK, and al. Lifestyle, diabetes, and cardiovascular risk factors 10 years after bariatric surgery. N Engl J Med. 2004 23; 351:2683-93.

35. Leonetti F, Capoccia D, Coccia F, Casella G, Baglio G, Paradiso F, et al. Obesity, Type 2 Diabetes Mellitus, and Other Comorbidities: A Prospective Cohort Study of Laparoscopic Sleeve Gastrectomy vs Medical Treatment. Arch Surg. 2012; 147:694-700.

36. Puzziferri N, Roshek TB, Mayo HG, Gallagher R, Belle SH, Livingston EH. Long-term Follow-up after Bariatric Surgery: A Systematic Review. JAMA. 2014; 312 :934.

37. Müller-Stich BP, Senft JD, Warschkow R, Kenngott HG, Billeter AT, Vit G, et al. Surgical Versus Medical Treatment of Type 2 Diabetes Mellitus in Nonseverely Obese Patients: A Systematic Review and Meta-analysis. Ann Surg. 2015; 261:421- 9.

38. Arterburn DE, Olsen MK, Smith VA, Livingston EH, Van Scoyoc L, Yancy WS, et al. Association Between Bariatric Surgery and Long-term Survival. JAMA. 2015; 313:62.

39. Sjöström L. Review of the key results from the Swedish Obese Subjects (SOS) trial - a prospective controlled intervention study of bariatric surgery. J Intern Med. 2013; 273:219- 34 .

40. Czernichow S, Moszkowicz D, Szwarcensztein K, Emery C, Lafuma A, Gourmelen J, et al. Impact of Bariatric Surgery on the Medical Management and Costs of Obese Patients in France: an Analysis of a National Representative Claims Database. Obes Surg. 2015;25:986- 96.

41. Ganguly S,Tan HC, Lee PC, Tham KW. Metabolic bariatric surgery and type 2 diabetes mellitus: an endocrinologist's perspective. J Biomed Res. 015;105- 11.

42. P. Verhaeghe, A. Dhahri, Q. Qassemyar, J-M Regimbeau. Technique de la gastrectomie longitudinale («sleeve gastrectomy») par laparoscopie - EMC Techniques chirurgicales Appareil digestif [40-385]

43. Dimitriadis E, Daskalakis M, Kampa M, Peppe A, Papadakis JA, Melissas J. Alterations in Gut Hormones After Laparoscopic Sleeve Gastrectomy: A Prospective Clinical and Laboratory Investigational Study. Ann Surg. 2013; 257:647- 54.

44. Cohen R, Uzzan B, Bihan H, Khochtali I, Reach G, Catheline JM. Ghrelin Levels and Sleeve Gastrectomy in Super-Super-Obesity. Obes Surg. 2005; 15:1501- 2. 
45. Gagnière J, Slim K, Launay-Savary M-V, Raspado O, Flamein R, Chipponi J. Previous gastric banding increases morbidity and gastric leaks after laparoscopic sleeve gastrectomy for obesity. J Visc Surg. 2011; 148:e205-9.

46. Trastulli S, Desiderio J, Guarino S, Cirocchi R, Scalercio V, Noya G, et al. Laparoscopic sleeve gastrectomy compared with other bariatric surgical procedures: a systematic review of randomized trials. Surg Obes Relat Dis. 2013; 9:816- 29.

47. Sjöström L. Bariatric surgery and reduction in morbidity and mortality: experiences from the SOS study. Int J Obes. 2008; 32:S93- 7.

48. Péquignot A, Dhahri A, Verhaeghe P, Desailloud R, Lalau J-D, Regimbeau J-M. Efficiency of laparoscopic sleeve gastrectomy on metabolic syndrome disorders: Two years results. J Visc Surg. 2012;149:e350- 5.

49. Lazzati A, Audureau É, Hemery F, Azoulay D, Iannelli A. Mortalité après chirurgie bariatrique sur 140000 patients. J Chir Viscérale. 2014;151:A7.

50. Melissas J, Koukouraki S, Askoxylakis J, Stathaki M, Daskalakis M, Perisinakis K, et al. Sleeve Gastrectomy — A Restrictive Procedure? Obes Surg. 2007; 17:57- 62.

51. Lalor PF, Tucker ON, Szomstein S, Rosenthal RJ. Complications after laparoscopic sleeve gastrectomy. Surg Obes Relat Dis. 2008;4:33- 8.

52. Aurora AR, Khaitan L, Saber AA. Sleeve gastrectomy and the risk of leak: a systematic analysis of 4,888 patients. Surg Endosc. 2012; 26:1509- 15.

53. Chang P-C, Tai C-M, Hsin M-C, Hung C-M, Huang IY-W, Huang C-K. Surgical standardization to prevent gastric stenosis after laparoscopic sleeve gastrectomy: a case series. Surg Obes Relat Dis 2016.

54. Parikh A, Alley JB, Peterson RM, Harnisch MC, Pfluke JM, Tapper DM, et al. Management options for symptomatic stenosis after laparoscopic vertical sleeve gastrectomy in the morbidly obese. Surg Endosc. 2012; 26:738- 46.

55. Fuks D, Verhaeghe P, Brehant O, Sabbagh C, Dumont F, Riboulot M, et al. Results of laparoscopic sleeve gastrectomy: A prospective study in 135 patients with morbid obesity. Surgery.2009; 145:106- 13.

56. Rebibo L, Dhahri A, Verhaeghe P, Regimbeau J-M. Early gastric fistula after laparoscopic sleeve gastrectomy: Surgical management. J Visc Surg. 2012; 149:e319- 24.

57. Moon RC, Shah N, Teixeira AF, Jawad MA. Management of staple line leaks following sleeve gastrectomy. Surg Obes Relat Dis. 2015; 11:54- 9.

58. Bruce J, Krukowski ZH, Al-Khairy G, Russell EM, Park KG. Systematic review of the definition and measurement of anastomotic leak after gastrointestinal surgery. Br J Surg. 2001; 88:1157-68.

59. Randal S Baker, James Foote, Paul Kemmeter, Randall Brady, Todd Vroegop, Matt Serveld. The science of stapling and leaks. Obesity Surgery. 2004, Volume 14,Issue 10, pp 1290-1298 
60. Walsh C, Karmali S Endoscopic management of bariatric complications: A review and update. World J Gastrointest Endosc. 2015 16; 7:518-23

61. Murino A, Arvanitakis M, Le Moine O, Blero D, Devière J, Eisendrath P. Effectiveness of Endoscopic Management Using Self-Expandable Metal Stents in a Large Cohort of Patients with Post-bariatric Leaks. Obes Surg. 2015; 25:1569- 76.

62. Puig CA, Waked TM, Baron TH, Wong Kee Song LM, Gutierrez J, Sarr MG. The role of endoscopic stents in the management of chronic anastomotic and staple line leaks and chronic strictures after bariatric surgery. Surg Obes Relat Dis. 2014; 10:613- 7.

63. Joo MK. Endoscopic Approach for Major Complications of Bariatric Surgery. Clin Endosc. $302017 ; 50: 31-41$.

64. Southwell T, Lim TH, Ogra R. Endoscopic Therapy for Treatment of Staple Line Leaks PostLaparoscopic Sleeve Gastrectomy (LSG): Experience from a Large Bariatric Surgery Centre in New Zealand. Obes Surg. 2016; 26:1155- 62.

65. Galloro G, Magno L, Musella M, Manta R, Zullo A, Forestieri P. A novel dedicated endoscopic stent for staple-line leaks after laparoscopic sleeve gastrectomy: a case series. Surg Obes Relat Dis. 2014; 10:607- 11.

66. Almadi MA. Fatal aortoesophageal fistula bleeding after stenting for a leak post sleeve gastrectomy. World J Gastrointest Surg. 2013; 5:337.

67. Simon F, Siciliano I, Gillet A, Castel B, Coffin B, Msika S. Gastric Leak After Laparoscopic Sleeve Gastrectomy: Early Covered Self-Expandable Stent Reduces Healing Time. Obes Surg. 2013; 23:687-92.

68. Vilallonga R, Himpens J, Bosch B, van de Vrande S, Bafort J. Role of Percutaneous Glue Treatment After Persisting Leak After Laparoscopic Sleeve Gastrectomy. Obes Surg. 2016;26 :1378- 83 .

69. Surace M, Mercky P, Demarquay J-F, Gonzalez J-M, Dumas R, Ah-Soune P, et al. Endoscopic management of GI fistulae with the over-the-scope clip system (with video). Gastrointest Endosc.2011; 74:1416-9.

70. Aly A, Lim HK. The Use of Over the Scope Clip (OTSC) Device for Sleeve Gastrectomy Leak. J Gastrointest Surg. 2013; 17 :606-8.

71. Mennigen R, Colombo-Benkmann M, Senninger N, Laukoetter M. Endoscopic Closure of Postoperative Gastrointestinal Leakages and Fistulas with the Over-the-Scope Clip (OTSC). J Gastrointest Surg. 2013; 17:1058- 65.

72. Shehab HM, Hakky SM, Gawdat KA. An Endoscopic Strategy Combining Mega Stents and Over-The-Scope Clips for the Management of Post-Bariatric Surgery Leaks and Fistulas (with video). Obes Surg.2016;26:941- 8.

73. Mercky P, Gonzalez J-M, Aimore Bonin E, Emungania O, Brunet J, Grimaud J-C, et al. Usefulness of over-the-scope clipping system for closing digestive fistulas: OTSC for closing digestive fistulas. Dig Endosc. 2015; 27:18- 24. 
74. Conio M, Blanchi S, Repici A, Bastardini R, Marinari G. Use of an over-the-scope clip for endoscopic sealing of a gastric fistula after sleeve gastrectomy. Endoscopy. 2010; 42:E71 - 2.

75. Bartoli E, Rebibo L, Robert B, Fumery M, Delcenserie R, Regimbeau J-M. Efficacy of the double-pigtail stent as a conservative treatment for grade B pancreatic fistula after pancreatoduodenectomy with pancreatogastric anastomosis. Surg Endosc. 2014;28:1528- 34.

76. Rebibo L, Bartoli E, Dhahri A, Cosse C, Robert B, Brazier F, et al. Persistent gastric fistula after sleeve gastrectomy: an analysis of the time between discovery and reoperation. Surg Obes Relat Dis. 2016; 12 :84- 93.

77. Gagner M, Deitel M, Erickson AL, Crosby RD. Survey on laparoscopic sleeve gastrectomy (LSG) at the Fourth International Consensus Summit on Sleeve Gastrectomy. Obes Surg. 2013; 23:2013- 7 .

78. Pequignot A, Fuks D, Verhaeghe P, Dhahri A, Brehant O, Bartoli E, et al. Is There a Place for Pigtail Drains in the Management of Gastric Leaks After Laparoscopic Sleeve Gastrectomy? Obes Surg. 2012;22:712- 20.

79. Donatelli G, Dumont J-L, Cereatti F, Ferretti S, Vergeau BM, Tuszynski T, et al. Treatment of Leaks Following Sleeve Gastrectomy by Endoscopic Internal Drainage (EID). Obes Surg. 2015;25:1293- 301.

80. Bouchard S, Eisendrath P, Toussaint E, Le Moine O, Lemmers A, Arvanitakis M, et al. Transfistulary endoscopic drainage for post-bariatric abdominal collections communicating with the upper gastrointestinal tract. Endoscopy. 2016; 48:809- 16.

81. Rebibo L, Hakim S, Brazier F, Dhahri A, Cosse C, Regimbeau J-M. New endoscopic technique for the treatment of large gastric fistula or gastric stenosis associated with gastric leaks after sleeve gastrectomy. Surg Obes Relat Dis. 2016; 12:1577- 84.

82. Genser L, Pattou F, Caiazzo R. Splenic abscess with portal venous gas caused by intrasplenic migration of an endoscopic double pigtail drain as a treatment of post-sleeve gastrectomy fistula. Surg Obes Relat Dis. 2016;12:e1 - 3.

83. Donatelli G, Airinei G, Poupardin E, Tuszynski T, Wind P, Benamouzig R, et al. Doublepigtail stent migration invading the spleen: rare potentially fatal complication of endoscopic internal drainage for sleeve gastrectomy leak. Endoscopy. 2016;48:E74-5. 


\section{Prise en charge endoscopique des fistules gastriques après sleeve gastrectomy par prothèses double queue}

de cochon : Technique BARTOLI.

Introduction. La fistule gastrique (FG) après sleeve gastrectomy (SG) est la principale complication postopératoire précoce, présente dans $2.5 \%$ des cas. L'objectif de cette étude était d'évaluer l'efficacité du drainage endoscopique de ces fistules par mise en place de prothèses double queue de cochon (PDQC).

Patients et méthode. Entre janvier 2010 et avril 2016 (PT), les patients ayant une FG après SG et ayant bénéficié de la pose de PDQC lors de la première endoscopie ont été inclus. L'objectif principal était l'évaluation de l'efficacité sur la cicatrisation des FG post SG par la pose de PDQC pour les périodes PT, P1 (décembre 2009-décembre 2013) et P2 (janvier 2014-mars 2016). Les objectifs secondaires étaient l'évaluation, pour les périodes PT, P1 et P2, de l'efficacité du traitement endoscopique, du délai de guérison de la FG, du taux de complication, de la tolérance clinique, du nombre et de la durée d'hospitalisation conventionnelle et en réanimation et du taux de mortalité.

Résultats. 82 patients ont été pris en charge, 34 lors de P1 et 48 lors de P2. Le taux d'efficacité par la mise en place de PDQC était de 79.2\% $(\mathrm{n}=65)$ pour PT, $70.5 \%(\mathrm{n}=24)$ pour P1 et $85.1 \%(\mathrm{n}=41)$ pour P2. Le taux d'efficacité de tous les traitements endoscopiques confondus était de 97.1\% pour P1 et de 97.9\%pour P2. La tolérance, le taux de migration symptomatique et le taux de perforation étaient notés respectivement à $97.6 \%$ $(\mathrm{n}=80), 9.7 \%(\mathrm{n}=8)$ et $3.6 \%(\mathrm{n}=3)$. La durée médiane de guérison de la FG était de 58 jours (IQR 46-76) pour PT et 48 jours (IQR 42.25-69) pour P2. La durée médiane d'hospitalisation totale et le taux d'hospitalisation en réanimation étaient, respectivement pour PT et P2, de 18.5 jours (IQR 13-31) et 11.5 jours (IQR 11.75-26), $34.1 \%(n=28)$ e $20.8 \%(n=10)$. Aucun décès n'était constaté.

Conclusion. La prise en charge des FG après SG par la technique Bartoli est efficace, bien tolérée avec une faible morbidité.

Mots clefs: fistule gastrique, sleeve gastrectomy, PDQC, endoscopie, chirurgie bariatrique.

\section{Endoscopic management of gastric leaks after laparoscopic sleeve gastrectomy, by internal drainage with double pigtail stent: BARTOLI}

Background: Gastric leak (GL) after sleeve gastrectomy (SG) is the main early postoperative complication, reported in about $2.5 \%$ of patients. There is no consensus on the management of these fistula. We aimed at evaluate the efficacy and safety of internal endoscopic drainage of GL after SG by double pigtail stent (DPS)

Patients and method. Between January 2010 and April 2016 (PT), patients with GL after SG who had received PDQC during the first endoscopy were included. The main objective was to evaluate the efficacy of healing GL after SG for PT, P1 (December 2009-December 2013) and P2 (January 2014-March 2016). Secondary objectives were the evaluation for PT, P1 and P2, the efficacy of all endoscopic treatment, GL healing time, complication rate, clinical tolerance, number and duration of conventional and intensive care hospitalization, and the mortality rate.

Results. 82 patients were treated, 34 at P1 and 48 at P2. The efficacy of PDQC treatment was 79.2\% $(\mathrm{n}=65)$ for PT, 70.5\% $(n=24)$ for P1 and 85.1\% $(n=41)$ for P2. The efficacy of all endoscopic treatments was $97.1 \%$ for $\mathrm{P} 1$ and $97.9 \%$ for P2. Tolerance, symptomatic migration rate and perforation rate were $97.6 \%(\mathrm{n}=80), 9.7 \%$ $(n=8)$ and $3.6 \%(n=3)$. The median healing time was 58 days (IQR 46-76) for PT and 48 (IQR 42.25-69) days for P2. The median total hospitalization were 18.5 days (IQR 13-31) and 11.5 days (IQR 11.75-26), and intensive care hospitalization rate was $34.1 \%(n=28)$ and $20.8 \%(n=10)$. No deaths were reported.

Conclusion. The management of GL by the Bartoli technique is effective, well tolerated with low morbidity.

Key words: gastric leak, sleeve gastrectomy, double pigtail stent, endoscopy, bariatric surgery. 